\title{
On Uncertainty Principle for Quaternionic Linear Canonical Transform
}

\author{
Kit Ian Kou, ${ }^{1}$ Jian-Yu Ou, ${ }^{1}$ and Joao Morais ${ }^{2}$ \\ ${ }^{1}$ Department of Mathematics, Faculty of Science and Technology, University of Macau, Macau \\ ${ }^{2}$ Center for Research and Development in Mathematics and Applications, Department of Mathematics, University of Aveiro, Portugal
}

Correspondence should be addressed to Kit Ian Kou; kikou@umac.mo

Received 9 November 2012; Revised 20 March 2013; Accepted 20 March 2013

Academic Editor: Natig M. Atakishiyev

Copyright (C) 2013 Kit Ian Kou et al. This is an open access article distributed under the Creative Commons Attribution License, which permits unrestricted use, distribution, and reproduction in any medium, provided the original work is properly cited.

We generalize the linear canonical transform (LCT) to quaternion-valued signals, known as the quaternionic linear canonical transform (QLCT). Using the properties of the LCT we establish an uncertainty principle for the QLCT. This uncertainty principle prescribes a lower bound on the product of the effective widths of quaternion-valued signals in the spatial and frequency domains. It is shown that only a $2 \mathrm{D}$ Gaussian signal minimizes the uncertainty.

\section{Introduction}

The classical uncertainty principle of harmonic analysis states that a nontrivial function and its Fourier transform (FT) cannot both be sharply localized. The uncertainty principle plays an important role in signal processing [1-11] and physics [1221]. In quantum mechanics an uncertainty principle asserts that one cannot make certain of the position and velocity of an electron (or any particle) at the same time. That is, increasing the knowledge of the position decreases the knowledge of the velocity or momentum of an electron. In quaternionic analysis some papers combine the uncertainty relations and the quaternionic Fourier transform (QFT) [22-24].

The QFT plays a vital role in the representation of (hypercomplex) signals. It transforms a real (or quaternionic) $2 \mathrm{D}$ signal into a quaternion-valued frequency domain signal. The four components of the QFT separate four cases of symmetry into real signals instead of only two as in the complex FT. In [25] the authors used the QFT to proceed color image analysis. The paper [26] implemented the QFT to design a color image digital watermarking scheme. The authors in [27] applied the QFT to image preprocessing and neural computing techniques for speech recognition. Recently, certain asymptotic properties of the QFT were analyzed and straightforward generalizations of classical Bochner-Minlos theorems to the framework of quaternionic analysis were derived $[28,29]$. In this paper, we study the uncertainty principle for the QLCT and the generalization of the QFT to the Hamiltonian quaternionic algebra.

The classical LCT being a generalization of the FT, was first proposed in the 1970s by Collins [30] and Moshinsky and Quesne [31]. It is an effective processing tool for chirp signal analysis, such as the parameter estimation, sampling progress for nonbandlimited signals with nonlinear Fourier atoms [32], and the LCT filtering [33-35]. The windowed LCT [36], with a local window function, can reveal the local LCT-frequency contents, and it enjoys high concentrations and eliminates cross terms. The analogue of the Poisson summation formula, sampling formulas, series expansions, Paley-Wiener theorem, and uncertainly relations is studied in $[36,37]$. In view of numerous applications, one is particularly interested in higher-dimensional analogues to Euclidean space. The LCT was first extended to the Clifford analysis setting in [38]. It was used to study the generalized prolate spheroidal wave functions and their connection to energy concentration problems [38]. In the present work, we study the QLCT which transforms a quaternionic 2D signal into a quaternion-valued frequency domain signal. Some important properties of the QLCT are analyzed. An uncertainty principle for the QLCT is established. This uncertainty principle prescribes a lower bound on the product of the effective widths of quaternion-valued signals in the spatial and frequency domains. To the best of our knowledge, the study of a Heisenberg-type uncertainty principle for the 
QLCT has not been carried out yet. The results in this paper are new in the literature. The main motivation of the present study is to develop further general numerical methods for differential equations and to investigate localization theorems for summation of Fourier series in the quaternionic analysis setting. Further investigations and extensions of this topic will be reported in a forthcoming paper.

The paper is organized as follows. Section 2 gives a brief introduction to some general definitions and basic properties of quaternionic analysis. The LCT of $2 \mathrm{D}$ quaternionic signal is introduced and studied in Section 3. Some important properties such as Parseval's and inversion theorems are obtained. In Section 4, we introduce and discuss the concept of QLCT and demonstrate some important properties that are necessary to prove the uncertainty principle for the QLCT. The classical Heisenberg uncertainty principle is generalized for the QLCT in Section 5. This principle prescribes a lower bound on the product of the effective widths of quaternionvalued signals in the spatial and frequency domains. Some conclusions are drawn in Section 6.

\section{Preliminaries}

The quaternionic algebra was invented by Hamilton in 1843

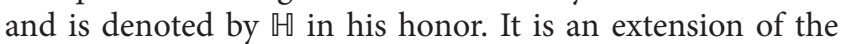
complex numbers to a 4D algebra. Every element of $\mathbb{W}$ is a linear combination of a real scalar and three orthogonal imaginary units (denoted, resp., by $\mathbf{i}, \mathbf{j}$, and $\mathbf{k}$ ) with real coefficients

$$
\mathbb{H}:=\left\{q=q_{0}+\mathbf{i} q_{1}+\mathbf{j} q_{2}+\mathbf{k} q_{3} \mid q_{0}, q_{1}, q_{2}, q_{3} \in \mathbb{R}\right\},
$$

where the elements $\mathbf{i}, \mathbf{j}$, and $\mathbf{k}$ obey Hamilton's multiplication rules

$$
\begin{aligned}
& \mathbf{i}^{2}=\mathbf{j}^{2}=\mathbf{k}^{2}=-1 ; \quad \mathbf{i j}=-\mathbf{j i}=\mathbf{k}, \\
& \mathbf{j k}=-\mathbf{k} \mathbf{j}=\mathbf{i}, \quad \mathbf{k} \mathbf{i}=-\mathbf{i} \mathbf{k}=\mathbf{j} .
\end{aligned}
$$

For every quaternionic number $q=q_{0}+q, q=\mathbf{i} q_{1}+\mathbf{j} q_{2}+\mathbf{k} q_{3}$, the scalar and nonscalar parts of $q$ are defined as $\operatorname{Sc}(q):=q_{0}$ and $\operatorname{NSc}(q):=q$, respectively.

Every quaternion $q=q_{0}+q$ has a quaternionic conjugate $\bar{q}=q_{0}-\underline{q}$. This leads to a norm of $q \in \mathbb{H}$ defined as

$$
|q|:=\sqrt{q \bar{q}}=\sqrt{q_{0}^{2}+q_{1}^{2}+q_{2}^{2}+q_{3}^{2}} .
$$

Let $|q|$ and $\theta(\in \mathbb{R})$ be polar coordinates of the point $\left(q_{0}, q\right) \in$ $\mathbb{W}$ that corresponds to a nonzero quaternion $q=q_{0}+q . q$ can be written in polar form as

$$
q=|q|(\cos \theta+\underline{e} \sin \theta),
$$

where $q_{0}=|q| \cos \theta,|q|=|q| \sin \theta, \theta=\arctan \left(|q| / q_{0}\right)$, and $\underline{e}=q /|q|$. If $q \equiv 0$, the coordinate $\theta$ is undefined; so it is always understood that $q \neq 0$ whenever $\theta=\arg q$ is discussed.

The symbol $e^{\underline{e} \theta}$, or $\exp (\underline{e \theta})$, is defined by means of an infinite series (or Euler's formula) as

$$
e^{\underline{e} \theta}:=\sum_{n=0}^{\infty} \frac{(\underline{e} \theta)^{n}}{n !}=\cos \theta+\underline{e} \sin \theta
$$

where $\theta$ is to be measured in radians. It enables us to write the polar form (4) in exponential form more compactly as

$$
q=|q| e^{\underline{\underline{e}} \theta}=|q| \exp \left(\frac{\underline{q}}{|\underline{q}|} \arctan \left(\frac{|\underline{q}|}{q_{0}}\right)\right) .
$$

Quaternions can be used for three- or four-entry vector analyses. Recently, quaternions have also been used for color image analysis. For $q=q_{0}+\mathbf{i} q_{1}+\mathbf{j} q_{2}+\mathbf{k} q_{3} \in \mathbb{H}$, we can use $q_{1}, q_{2}$, and $q_{3}$ to represent, respectively, the $R, G$, and $B$ values of a color image pixel and set $q_{0}=0$.

For $p=1$ and 2 , the quaternion modules $L^{p}\left(\mathbb{R}^{2} ; \mathbb{H}\right)$ are defined as

$$
\begin{aligned}
L^{p}\left(\mathbb{R}^{2} ; \mathbb{U}\right):= & \left\{f \mid f: \mathbb{R}^{2} \longrightarrow \mathbb{H},\|f\|_{L^{p}\left(\mathbb{R}^{2} ; \mathbb{G}\right)}\right. \\
& \left.:=\int_{\mathbb{R}^{2}}\left|f\left(x_{1}, x_{2}\right)\right|^{p} d x_{1} d x_{2}<\infty\right\} .
\end{aligned}
$$

For two quaternionic signals $f, g \in L^{2}\left(\mathbb{R}^{2} ; \mathbb{H}\right)$ the quaternionic space can be equipped with a Hermitian inner product,

$$
\langle f, g\rangle_{L^{2}\left(\mathbb{R}^{2} ; \boxplus\right)}:=\int_{\mathbb{R}^{2}} f\left(x_{1}, x_{2}\right) \overline{g\left(x_{1}, x_{2}\right)} d x_{1} d x_{2},
$$

whose associated norm is

$$
\|f\|_{L^{2}\left(\mathbb{R}^{2} ; \mathbb{W}\right)}:=\left(\int_{\mathbb{R}^{2}}\left|f\left(x_{1}, x_{2}\right)\right|^{2} d x_{1} d x_{2}\right)^{1 / 2} .
$$

As a consequence of the inner product (9), we obtain the quaternionic Cauchy-Schwarz inequality

$$
\begin{aligned}
\left|\operatorname{Sc}\left(\langle f, g\rangle_{L^{2}\left(\mathbb{R}^{2} ; \mathbb{H}\right)}\right)\right| & \leq\left|\langle f, g\rangle_{L^{2}\left(\mathbb{R}^{2} ; \mathbb{H}\right)}\right| \\
& \leq\|f\|_{L^{2}\left(\mathbb{R}^{2} ; \mathbb{H}\right)}\|g\|_{L^{2}\left(\mathbb{R}^{2} ; \mathbb{W}\right)}
\end{aligned}
$$

for any $f, g \in L^{2}\left(\mathbb{R}^{2} ; \mathbb{H}\right)$.

In $[39,40]$ a Clifford-valued generalized function theory is developed. In the following, we adopt the definition that $T$ is called a tempered distribution, if $T$ is a continuous linear functional from $\mathbb{S}:=\mathbb{S}\left(\mathbb{R}^{2}\right)$ to $\mathbb{H}$, where $\mathbb{S}\left(\mathbb{R}^{2}\right)$ is the Schwarz class of rapidly decreasing functions. The set of all tempered distributions is denoted by $\mathbb{S}^{\prime}$. If $T \in \mathbb{S}^{\prime}$, we denote this value for a test function $\phi$ by writing

$$
T[\phi]:=\int_{\mathbb{R}} T\left(x_{1}, x_{2}\right) \phi\left(x_{1}, x_{2}\right) d x_{1} d x_{2},
$$

using square brackets. (In the literature one often sees the notation $\langle T, \phi\rangle$, but we shall avoid this, since it does not completely share the properties of the inner product.)

This is equivalent to the one defined in [39] using modules and enables us to define Fourier transforms on tempered distributions, by the formula

$$
\widehat{T}[\phi]=T[\widehat{\phi}], \quad \forall \phi \in \mathbb{S},
$$

which is just to perform Fourier transform

$$
\widehat{\phi}\left(\omega_{1}, \omega_{2}\right)=\int_{\mathbb{R}^{2}} \phi\left(x_{1}, x_{2}\right) e^{\mathbf{i}\left(x_{1} \omega_{1}+x_{2} \omega_{2}\right)} d x_{1} d x_{2}
$$


on each of the components of the distribution. We will use the following results:

$$
\begin{aligned}
& \widehat{1}\left(\omega_{1}, \omega_{2}\right)=(2 \pi)^{2} \delta\left(\omega_{1}, \omega_{2}\right), \\
& \left(\mathbf{i}^{-\widehat{|\alpha|} D^{\alpha}} \delta\right)\left(\omega_{1}, \omega_{2}\right)=\omega_{1}^{\alpha_{1}} \omega_{2}^{\alpha_{2},}
\end{aligned}
$$

where $\alpha=\left(\alpha_{1}, \alpha_{2}\right),|\alpha|=\alpha_{1}+\alpha_{2}, D^{\alpha}=\left(\partial / \partial x_{1}\right)^{\alpha_{1}}\left(\partial / \partial x_{2}\right)^{\alpha_{2}}$, and $\delta$ is the usual Dirac delta function.

In the following we introduce the LCT for $2 \mathrm{D}$ quaternionic signals.

\section{LCTs of 2D Quaternionic Signals}

The LCT was first introduced in the $70 \mathrm{~s}$ and is a fourparameter class of linear integral transform, which includes among its many special cases the FT, the fractional Fourier transform (FRFT), the Fresnel transform, the Lorentz transform, and scaling operations. In a way, the LCT has more degrees of freedom and is more flexible than the FT and the FRFT, but with similar computation cost as the conventional FT [41]. Due to the mentioned advantages, it is natural to generalize the classical LCT to the quaternionic algebra.

3.1. Definition. Using the definition of the LCT $[33,42]$, we extend the LCT to the 2D quaternionic signals. Let us define the left-sided and right-sided LCTs of 2D quaternionic signals.

Definition 1 (left-sided and right-sided LCTs). Let $A_{i}=$ $\left[\begin{array}{ll}a_{i} & b_{i} \\ c_{i} & d_{i}\end{array}\right] \in \mathbb{R}^{2 \times 2}$ be a matrix parameter such that $\operatorname{det}\left(A_{i}\right)=1$, for $i=1,2$. The left-sided and right-sided LCTs of 2D quaternionic signals $f \in L^{1}\left(\mathbb{R}^{2} ; \mathfrak{H}\right)$ are defined by

$$
\begin{aligned}
& L_{l}^{\mathbf{i}}(f)\left(u_{1}, x_{2}\right):= \begin{cases}\frac{1}{\sqrt{\mathbf{i} 2 \pi b_{1}}} \int_{\mathbb{R}} e^{\mathbf{i}\left(\left(a_{1} / 2 b_{1}\right) x_{1}^{2}-\left(1 / b_{1}\right) x_{1} u_{1}+\left(d_{1} / 2 b_{1}\right) u_{1}^{2}\right)} f\left(x_{1}, x_{2}\right) d x_{1}, & b_{1} \neq 0 ; \\
\sqrt{d_{1}} e^{\mathbf{i}\left(c_{1} d_{1} / 2\right) u_{1}^{2}} f\left(d_{1} u_{1}, x_{2}\right), & b_{1}=0,\end{cases} \\
& L_{r}^{\mathbf{j}}(f)\left(x_{1}, u_{2}\right):= \begin{cases}\int_{\mathbb{R}} f\left(x_{1}, x_{2}\right) \frac{1}{\sqrt{\mathbf{j} 2 \pi b_{2}}} e^{\mathbf{j}\left(\left(a_{2} / 2 b_{2}\right) x_{2}^{2}-\left(1 / b_{2}\right) x_{2} u_{2}+\left(d_{2} / 2 b_{2}\right) u_{2}^{2}\right)} d x_{2}, & b_{2} \neq 0 ; \\
f\left(x_{1}, d_{2} u_{2}\right) \sqrt{d_{2}} e^{\mathbf{j}\left(c_{2} d_{2} / 2 u_{2}^{2}\right)}, & b_{2}=0,\end{cases}
\end{aligned}
$$

respectively.

Note that, for $b_{i}=0(i=1,2)$, the LCT of a signal is essentially a chirp multiplication and it is of no particular interest for our objective in this work. Hence, without loss of generality, we set $b_{i} \neq 0$ in the following sections unless stated otherwise. Therefore

$$
\begin{aligned}
& L_{l}^{\mathbf{i}}(f)\left(u_{1}, x_{2}\right)=\int_{\mathbb{R}} K_{A_{1}}^{\mathbf{i}}\left(x_{1}, u_{1}\right) f\left(x_{1}, x_{2}\right) d x_{1}, \quad b_{1} \neq 0, \\
& L_{r}^{\mathbf{j}}(f)\left(x_{1}, u_{2}\right)=\int_{\mathbb{R}} f\left(x_{1}, x_{2}\right) K_{A_{2}}^{\mathbf{j}}\left(x_{2}, u_{2}\right) d x_{2}, \quad b_{2} \neq 0,
\end{aligned}
$$

where the kernel functions

$$
\begin{aligned}
& K_{A_{1}}^{\mathbf{i}}\left(x_{1}, u_{1}\right):=\frac{1}{\sqrt{\mathbf{i} 2 \pi b_{1}}} e^{\mathbf{i}\left(\left(a_{1} / 2 b_{1}\right) x_{1}^{2}-\left(1 / b_{1}\right) x_{1} u_{1}+\left(d_{1} / 2 b_{1}\right) u_{1}^{2}\right)}, \\
& K_{A_{2}}^{\mathbf{j}}\left(x_{2}, u_{2}\right):=\frac{1}{\sqrt{\mathbf{j} 2 \pi b_{2}}} e^{\mathbf{i}\left(\left(a_{2} / 2 b_{2}\right) x_{2}^{2}-\left(1 / b_{2}\right) x_{2} u_{2}+\left(d_{2} / 2 b_{2}\right) u_{2}^{2}\right)},
\end{aligned}
$$

respectively.

3.2. Properties. The following proposition summarizes some important properties of the kernel functions $K_{A_{1}}^{\mathbf{i}}\left(\right.$ and $K_{A_{2}}^{\mathbf{j}}$ ) of the left-sided (and right-sided) LCTs which will be useful to study the properties of LCTs, such as the Plancherel theorem.
Proposition 2. Let the kernel function $K_{A}$ be defined by (19) or (20). Then

(i) $K_{A}(-x, \omega)=K_{A}(x,-\omega)$;

(ii) $K_{A}(-x,-\omega)=K_{A}(x, \omega)$;

(iii) $\overline{K_{A}(x, \omega)}=K_{B}(x, \omega)$, where $B=\left(\begin{array}{cc}a & -b \\ -c & d\end{array}\right)$;

(iv) $\int_{\mathbb{R}} K_{A_{1}}(x, \omega) K_{A_{2}}(\omega, y) d \omega=K_{A_{1} A_{2}}(x, y)$, where

The proofs of properties (i) to (iii) follow from definitions (19) and (20). The proof of property (iv) can be found in [33, 35].

Note that some properties of the LCT for 2D quaternionic signals follow from the one-dimensional case [35, 42].

Proposition 3. Let $L_{A_{i}}(f)(i=1,2)$ be defined by $L_{l}^{\mathbf{i}}$ in (17) or $L_{r}^{\mathbf{j}}$ in (18), respectively. If $f, g \in L^{1} \cap L^{2}\left(\mathbb{R}^{2} ; \mathbb{H}\right)$, then the following properties hold.

(i) Additivity:

$$
\begin{aligned}
& L_{A_{2}}\left(L_{A_{1}}\right)(f)=L_{A_{2} A_{1}}(f), \quad \text { for } L_{A_{i}}:=L_{l}^{\mathbf{i}}, \\
& L_{A_{2}}\left(L_{A_{1}}\right)(f)=L_{A_{1} A_{2}}(f), \quad \text { for } L_{A_{i}}:=L_{r}^{\mathbf{j}} .
\end{aligned}
$$


(ii) Reversibility:

$$
\begin{aligned}
& L_{A_{i}{ }^{-1}}\left(L_{A_{i}}\right)(f)=f, \quad \text { for } L_{A_{i}}:=L_{l}^{\mathbf{i}}, \\
& L_{A_{i}{ }^{-1}}\left(L_{A_{i}}\right)(f)=f, \quad \text { for } L_{A_{i}}:=L_{r}^{\mathbf{j}} .
\end{aligned}
$$

(iii) Plancherel Theorem (right-sided LCT): If $f, g \in \mathbb{S}$, then

$$
\langle f, g\rangle_{L^{2}\left(\mathbb{R}^{2} ; \mathbb{H}\right)}=\left\langle L_{r}^{\mathbf{j}}(f), L_{r}^{\mathbf{j}}(g)\right\rangle_{L^{2}\left(\mathbb{R}^{2} ; \mathbb{H}\right)} .
$$
is,

In particular, with $f=g$, we get the Parseval theorem; that

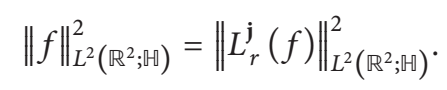

Proof. By Fubini's theorem, property (iv) of Proposition 2 establishes the additivity property (i) of left-sided LCTs,

$$
\begin{aligned}
& L_{A_{2}}\left(L_{A_{1}}(f)\left(u_{1}, x_{2}\right)\right)\left(u_{1}, x_{2}\right) \\
& \quad=\int_{\mathbb{R}} K_{A_{2}}^{\mathbf{i}}\left(u_{1}, y_{1}\right) L_{A_{1}}(f)\left(u_{1}, x_{2}\right) d u_{1} \\
& =\int_{\mathbb{R}}\left(\int_{\mathbb{R}} K_{A_{2}}^{\mathbf{i}}\left(u_{1}, y_{1}\right) K_{A_{1}}^{\mathbf{i}}\left(x_{1}, u_{1}\right) d u_{1}\right) f\left(x_{1}, x_{2}\right) d x_{1} \\
& =L_{A_{2} A_{1}}(f)\left(y_{1}, x_{2}\right) .
\end{aligned}
$$

The proof of the right-sided LCT $L_{A_{i}}:=L_{r}^{\mathbf{j}}$ is similar.

Reversibility property (ii) is an immediate consequence of additivity property (i) once we observe that $A_{1}=A_{i}$ and $A_{2}=A_{i}^{-1}$.

To verify property (iii), applying Fubini's theorem, it suffices to see that

$$
\begin{aligned}
& \left\langle L_{r}^{\mathbf{j}}(f), L_{r}^{\mathbf{j}}(g)\right\rangle_{L^{2}\left(\mathbb{R}^{2} ; \mathbb{E}\right)} \\
& =\int_{\mathbb{R}^{2}} L_{r}^{\mathbf{j}}(f)\left(u_{1}, x_{2}\right) \overline{L_{r}^{\mathbf{j}}(g)\left(u_{1}, x_{2}\right)} d u_{1} d x_{2} \\
& =\int_{\mathbb{R}^{4}} f\left(x_{1}, x_{2}\right) K_{A_{1}}^{\mathbf{j}}\left(x_{1}, u_{1}\right) \\
& \times \overline{K_{A_{1}}^{\mathbf{j}}\left(y_{1}, u_{1}\right)} \overline{g\left(y_{1}, x_{2}\right)} d u_{1} d x_{1} d y_{1} d x_{2} \\
& =\frac{1}{2 \pi b_{1}} \int_{\mathbb{R}^{4}} f\left(x_{1}, x_{2}\right) e^{\mathbf{j}\left(a_{1} / 2 b_{1}\right)\left(x_{1}^{2}-y_{1}^{2}\right)} \\
& \times e^{-\mathbf{j}\left(1 / b_{1}\right) u_{1}\left(x_{1}-y_{1}\right)} \overline{g\left(y_{1}, x_{2}\right)} d u_{1} d x_{1} d y_{1} d x_{2} \\
& =\int_{\mathbb{R}^{3}} f\left(x_{1}, x_{2}\right) e^{\mathbf{j}\left(a_{1} / 2 b_{1}\right)\left(x_{1}^{2}-y_{1}^{2}\right)} \\
& \times \delta\left(y_{1}-x_{1}\right) \overline{g\left(y_{1}, x_{2}\right)} d x_{1} d y_{1} d x_{2} \\
& =\langle f, g\rangle_{L^{2}\left(\mathbb{R}^{2} ; \mathbb{H}\right)},
\end{aligned}
$$

where we have used (14).
Notice that the left-sided and right-sided LCTs of quaternionic signals are unitary operators on $L^{2}\left(\mathbb{R}^{2} ; \mathbb{H}\right)$. In signal analysis, it is interpreted in the sense that (right-sided) LCT of quaternionic signal preserves the energy of a signal.

Remark 4. Note that the Plancherel theorem is not valid for the two-sided or left-sided LCT of 2D quaternionic signal. For this reason, we study the right-sided LCT of $2 \mathrm{D}$ quaternionic signals in the following.

It is worth noting that when $A_{1}=A_{2}=\left[\begin{array}{cc}0 & 1 \\ -1 & 0\end{array}\right]$, the leftsided and right-sided LCTs of $f$ reduce to the left-sided and right-sided FTs of $f$. That is,

$$
\begin{aligned}
L_{l}^{\mathbf{i}}(f)\left(u_{1}, x_{2}\right) & =\frac{1}{\sqrt{2 \pi \mathbf{i}}} \int_{\mathbb{R}} e^{-\mathbf{i} x_{1} u_{1}} f\left(x_{1}, x_{2}\right) d x_{1} \\
& =\frac{1}{\sqrt{2 \pi \mathbf{i}}} F_{l}^{\mathbf{i}}(f)\left(u_{1}, x_{2}\right), \\
L_{r}^{\mathbf{j}}(f)\left(x_{1}, u_{2}\right) & =\frac{1}{\sqrt{2 \pi \mathbf{j}}} \int_{\mathbb{R}} f\left(x_{1}, x_{2}\right) e^{-\mathbf{j} x_{2} u_{2}} d x_{2} \\
& =\frac{1}{\sqrt{2 \pi \mathbf{j}}} F_{r}^{\mathbf{j}}(f)\left(x_{1}, u_{2}\right),
\end{aligned}
$$

respectively. Here

$$
\begin{aligned}
& F_{l}^{\mathbf{i}}(f)\left(u_{1}, x_{2}\right):=\int_{\mathbb{R}} e^{-\mathbf{i} x_{1} u_{1}} f\left(x_{1}, x_{2}\right) d x_{1}, \\
& F_{r}^{\mathbf{j}}(f)\left(x_{1}, u_{2}\right):=\int_{\mathbb{R}} f\left(x_{1}, x_{2}\right) e^{-\mathbf{j} x_{2} u_{2}} d x_{2}
\end{aligned}
$$

are the left-sided FT and right-sided FT of $f$, respectively.

We now formulate the linear canonical integral representation of a $2 \mathrm{D}$ quaternionic signal $f$.

Theorem 5 (linear canonical inversion theorem). Suppose that $f \in L^{1}\left(\mathbb{R}^{2} ; \mathbb{H}\right)$, that $f$ is continuous except for a finite number of finite jumps in any finite interval, and that $f(s, t)=$ $(1 / 2)(f(s, t+)+f(s, t-))$ for all $t$ and $s$. Then

$$
f\left(s, t_{0}\right)=\lim _{\alpha \rightarrow \infty} \int_{-\alpha}^{\alpha} L_{r}^{\mathbf{i}}(f)(s, \omega) K_{A^{-1}}^{\mathbf{i}}\left(\omega, t_{0}\right) d \omega
$$

for every $t_{0}$ and $s$ where $f$ has (generalized) left and right partial derivatives. In particular, if $f$ is piecewise smooth (i.e., continuous and with a piecewise continuous derivative), then the formula holds for all $t_{0}$ and uniformly in $s$.

Proof. Put

$$
I\left(s, t_{0} ; \alpha\right):=\int_{-\alpha}^{\alpha} L_{r}^{\mathbf{i}}(f)(s, \omega) K_{A^{-1}}^{\mathbf{i}}\left(\omega, t_{0}\right) d \omega
$$

and rewrite this expression by inserting the definition of $L_{r}^{\mathbf{i}}(f)$,

$I\left(s, t_{0} ; \alpha\right)$

$$
=\int_{-\alpha}^{\alpha}\left(\int_{\mathbb{R}} f(s, t) K_{A}^{\mathbf{i}}(t, \omega) d t\right) K_{A^{-1}}^{\mathbf{i}}\left(\omega, t_{0}\right) d \omega
$$




$$
\begin{aligned}
= & \int_{\mathbb{R}} \int_{-\alpha}^{\alpha} f(s, t) K_{A}^{\mathbf{i}}(t, \omega) K_{A^{-1}}^{\mathbf{i}}\left(\omega, t_{0}\right) d \omega d t \\
= & \int_{\mathbb{R}} f(s, t)\left[\frac{1}{2 \pi b} e^{\mathbf{i}(a / 2 b)\left(t^{2}-t_{0}^{2}\right)} \int_{-\alpha}^{\alpha} e^{\mathbf{i}(1 / b)\left(t_{0}-t\right) \omega} d \omega\right] d t \\
= & \frac{1}{4 \pi} e^{-\mathbf{i}(a / 2 b) t_{0}^{2}} \int_{\mathbb{R}} f(s, t)\left(e^{\mathbf{i}(a / 2 b) t^{2}} \frac{\sin \left((\alpha / b)\left(t_{0}-t\right)\right)}{t_{0}-t}\right) d t \\
= & \frac{1}{4 \pi} e^{-\mathbf{i}(a / 2 b) t_{0}^{2}} \int_{\mathbb{R}} f\left(s, t_{0}-u\right) \\
& \times\left(e^{\mathbf{i}(a / 2 b)\left(t_{0}-u\right)^{2}} \frac{\sin ((\alpha / b) u)}{u}\right) d u .
\end{aligned}
$$

Switching the order of integration is permitted, because the improper double integral is absolutely convergent over the strip $(t, \omega) \in \mathbb{R} \times[-\alpha, \alpha]$, and in the last step we have put $t_{0}-t=u$. Using the formula

$$
\int_{0}^{\infty} e^{\mathrm{i}(a / 2 b)\left(t_{0}-u\right)^{2}} \frac{\sin ((\alpha / b) u)}{u} d u=2 \pi e^{\mathrm{i}(a / 2 b) t_{0}^{2}}, \quad \text { for } \alpha, b>0,
$$

we can write

$$
\begin{aligned}
& \frac{1}{2 \pi} e^{-\mathrm{i}(a / 2 b) t_{0}^{2}} \int_{0}^{\infty} f\left(s, t_{0}-u\right) \\
& \quad \times\left(e^{\mathrm{i}(a / 2 b)\left(t_{0}-u\right)^{2}} \frac{\sin ((\alpha / b) u)}{u}\right) d u \\
& -f\left(s, t_{0}-\right) \\
& =\frac{1}{2 \pi} e^{-\mathrm{i}(a / 2 b) t_{0}^{2}} \int_{0}^{\infty}\left(f\left(s, t_{0}-u\right)-f\left(s, t_{0}-\right)\right) \\
& \quad \times\left(e^{\mathrm{i}(a / 2 b)\left(t_{0}-u\right)^{2}} \frac{\sin ((\alpha / b) u)}{u}\right) d u .
\end{aligned}
$$

Now let $\epsilon>0$ be given. Since we have assumed that $f \epsilon$ $L^{1}\left(\mathbb{R}^{2} ; \mathbb{W}\right)$, there exists a number $\beta$ such that

$$
\frac{1}{2 \pi} \int_{\beta}^{\infty}\left|f\left(s, t_{0}-u\right)\right| d u<\epsilon
$$

Changing the variable, we find that

$$
\begin{aligned}
& \int_{\beta}^{\infty} e^{\mathrm{i}(a / 2 b)\left(t_{0}-u\right)^{2}} \frac{\sin ((\alpha / b) u)}{u} d u \\
&=\int_{\alpha \beta / b}^{\infty} e^{\mathrm{i}(a / 2 b)\left(t_{0}-b x / \alpha\right)^{2}} \frac{\sin x}{x} d x \longrightarrow 0, \\
& \text { as } \frac{\alpha}{b} \longrightarrow \infty .
\end{aligned}
$$

The last integral in (33) can be split into three terms:

$$
\begin{gathered}
\frac{1}{2 \pi} e^{-\mathbf{i}(a / 2 b) t_{0}^{2}} \int_{0}^{\beta} \frac{f\left(s, t_{0}-u\right)-f\left(s, t_{0}-\right)}{u} \\
\times\left(e^{\mathbf{i}(a / 2 b)\left(t_{0}-u\right)^{2}} \sin ((\alpha / b) u)\right) d u \\
+\frac{1}{2 \pi} e^{-\mathbf{i}(a / 2 b) t_{0}^{2}} \int_{\beta}^{\infty} f\left(s, t_{0}-u\right) \\
\times\left(e^{\mathbf{i}(a / 2 b)\left(t_{0}-u\right)^{2}} \frac{\sin ((\alpha / b) u)}{u}\right) d u \\
-\frac{1}{2 \pi} e^{-\mathbf{i}(a / 2 b) t_{0}^{2}} f\left(s, t_{0}-\right) \\
\quad \times \int_{\beta}^{\infty}\left(e^{\mathbf{i}(a / 2 b)\left(t_{0}-u\right)^{2}} \frac{\sin ((\alpha / b) u)}{u}\right) d u \\
=I_{1}+I_{2}-I_{3} .
\end{gathered}
$$

The term $I_{3}$ tends to zero as $b>0$ and $\alpha \rightarrow \infty$, because of (35). The term $I_{2}$ can be estimated:

$$
\begin{aligned}
\left|I_{2}\right|=\mid \frac{1}{2 \pi} e^{-\mathbf{i}(a / 2 b) t_{0}^{2}} \int_{\beta}^{\infty} f\left(s, t_{0}-u\right) & \\
& \times\left(e^{\mathbf{i}(a / 2 b)\left(t_{0}-u\right)^{2}} \frac{\sin ((\alpha / b) u)}{u}\right) d u \mid \\
\leq & \frac{1}{2 \pi} \int_{\beta}^{\infty}\left|f\left(s, t_{0}-u\right)\right| d u \leq \epsilon .
\end{aligned}
$$

In the term $I_{1}$ we have the function $g(s, u)=\left(f\left(s, t_{0}-\right.\right.$ $\left.u)-f\left(s, t_{0}-\right)\right) / u$. This is continuous except for jumps in the interval $\mathbb{R} \times(0, \beta)$, and it has the finite limit $g(s, 0+)=$ $(\partial / \partial t) f_{L}\left(s, t_{0}\right)$ as $u \searrow 0$; this means that $g$ is bounded uniformly in $s$ and thus integrable on the interval. By the Riemann-Lebesgue lemma, we conclude that $I_{1} \rightarrow 0$ as $\beta \rightarrow \infty$. All this together gives, since $\epsilon$ can be taken as small as we wish,

$$
\begin{aligned}
& \frac{1}{2 \pi} e^{-\mathbf{i}(a / 2 b) t_{0}^{2}} \\
& \times \int_{0}^{\infty} f\left(s, t_{0}-u\right) \\
& \quad \times\left(e^{\mathrm{i}(a / 2 b)\left(t_{0}-u\right)^{2}} \frac{\sin ((\alpha / b) u)}{u}\right) d u \rightarrow f\left(s, t_{0}-\right), \\
& \text { as } \frac{\alpha}{b} \longrightarrow \infty .
\end{aligned}
$$

A parallel argument implies that the corresponding integral over $(-\infty, 0)$ tends to $f\left(s, t_{0}+\right)$ uniformly in $s$. Taking the mean value of these two results, we have completed the proof of the theorem. 
Remark 6. If $L_{r}^{\mathbf{i}}(f) \in L^{1}\left(\mathbb{R}^{2} ; \mathbb{H}\right)$, then (29) can be written as the absolutely convergent integral

$$
f\left(s, t_{0}\right)=\int_{\mathbb{R}} L_{r}^{\mathbf{i}}(f)(s, \omega) K_{A^{-1}}^{\mathbf{i}}\left(\omega, t_{0}\right) d \omega .
$$

The following lemma gives the relationship between the left-(right-) sided LCTs and Left-(right-) sided FTs of $f$.

Lemma 7. Let $A_{i}=\left[\begin{array}{ll}a_{i} & b_{i} \\ c_{i} & d_{i}\end{array}\right] \in \mathbb{R}^{2 \times 2}$ be a matrix parameter such that $\operatorname{det}\left(A_{i}\right)=1$, for $i=1,2$. Let $f \in L^{1}\left(\mathbb{R}^{2} ; \mathbb{U}\right)$; then one has

$$
\begin{aligned}
L_{l}^{\mathbf{i}}(f)\left(u_{1}, x_{2}\right)= & e^{\mathbf{i}\left(d_{1} / 2 b_{1}\right) u_{1}^{2}} F_{l}^{\mathbf{i}}\left(\frac{1}{\sqrt{\mathbf{i} 2 \pi b_{1}}} e^{\mathbf{i}\left(a_{1} / 2 b_{1}\right)(\cdot)^{2}} f\left(\cdot, x_{2}\right)\right) \\
& \times\left(\frac{u_{1}}{b_{1}}, x_{2}\right),
\end{aligned}
$$

$$
\begin{aligned}
L_{r}^{\mathbf{j}}(f) & \left(x_{1}, u_{2}\right) \\
= & \left(F_{r}^{\mathbf{j}}\left(f\left(x_{1}, \cdot\right) \frac{1}{\sqrt{\mathbf{j} 2 \pi b_{2}}} e^{\mathbf{j}\left(a_{2} / 2 b_{2}\right)(\cdot)^{2}}\right)\right. \\
& \left.\times\left(x_{1}, \frac{u_{2}}{b_{2}}\right)\right) e^{\mathbf{j}\left(d_{2} / 2 b_{2}\right) u_{2}^{2}} .
\end{aligned}
$$

Proof. By the definition of $L_{l}^{\mathbf{i}}(f)$ in (17), a direct computation shows that

$$
\begin{aligned}
& L_{l}^{\mathbf{i}}(f)\left(u_{1}, x_{2}\right) \\
&=\int_{\mathbb{R}} \frac{1}{\sqrt{\mathbf{i} 2 \pi b_{1}}} e^{\mathbf{i}\left(\left(a_{1} / 2 b_{1}\right) x_{1}^{2}-\left(1 / b_{1}\right) x_{1} u_{1}+\left(d_{1} / 2 b_{1}\right) u_{1}^{2}\right)} f\left(x_{1}, x_{2}\right) d x_{1} \\
&= e^{\mathbf{i}\left(d_{1} / 2 b_{1}\right) u_{1}^{2}} \\
& \times\left(\int_{\mathbb{R}} e^{-\mathbf{i} x_{1}\left(u_{1} / b_{1}\right)}\left(\frac{1}{\sqrt{\mathbf{i} 2 \pi b_{1}}} e^{\mathbf{i}\left(a_{1} / 2 b_{1}\right) x_{1}^{2}} f\left(x_{1}, x_{2}\right)\right) d x_{1}\right) \\
&= e^{\mathbf{i}\left(d_{1} / 2 b_{1}\right) u_{1}^{2}} F_{l}^{\mathbf{i}}\left(\frac{1}{\sqrt{\mathbf{i} 2 \pi b_{1}}} e^{\mathbf{i}\left(a_{1} / 2 b_{1}\right)(\cdot)^{2}} f\left(\cdot, x_{2}\right)\right)\left(\frac{u_{1}}{b_{1}}, x_{2}\right) .
\end{aligned}
$$

Similarly, by the definition of $L_{r}^{\mathbf{j}}(f)$ in (18), we obtain (41).

The LCT can be further generalized into the offset linear canonical transform (offset LCT) [33, 43, 44]. It has two extra parameters which represent the space and frequency offsets. The basic theories of the LCT have been developed including uncertainty principles $[20,45]$, convolution theorem $[42,46]$, the Hilbert transform [11, 47], sampling theory [32, 42], and discretization $[41,48,49]$, which enrich the theoretical system of the LCT. On the other hand, since the LCT has three free parameters, it is more flexible and has found many applications in radar system analysis, filter design, phase retrieval, pattern recognition, and many other areas $[35,42]$.

\section{QLCTs of 2D Quaternionic Signals}

4.1. Definition. This section leads to the quaternionic linear canonical transforms (QLCTs). Due to the noncommutative property of multiplication of quaternions, there are many different types of QLCTs: two-sided QLCTs, left-sided QLCTs, and right-sided QLCTs.

Definition 8 (two-sided QLCTs). Let $A_{i}=\left[\begin{array}{cc}a_{i} & b_{i} \\ c_{i} & d_{i}\end{array}\right] \in \mathbb{R}^{2 \times 2}$ be a matrix parameter such that $\operatorname{det}\left(A_{i}\right)=1, b_{i} \neq 0$ for $i=$ 1,2 . The two-sided QLCTs of signals $f \in L^{1}\left(\mathbb{R}^{2} ; \mathbb{H}\right)$ are the functions $\mathscr{L}^{\mathbf{i}, \mathbf{j}}(f): \mathbb{R}^{2} \rightarrow \mathbb{W}$ given by

$$
\begin{aligned}
& \mathscr{L}^{\mathbf{i}, \mathbf{j}}(f)\left(u_{1}, u_{2}\right) \\
& \quad:=\int_{\mathbb{R}^{2}} K_{A_{1}}^{\mathbf{i}}\left(x_{1}, u_{1}\right) f\left(x_{1}, x_{2}\right) K_{A_{2}}^{\mathbf{j}}\left(x_{2}, u_{2}\right) d x_{1} d x_{2},
\end{aligned}
$$

where $u_{1}, u_{2}=u_{1} \mathbf{e}_{1}+u_{2} \mathbf{e}_{2}$, with $K_{A_{1}}^{\mathbf{i}}\left(x_{1}, u_{1}\right)$ and $K_{A_{2}}^{\mathbf{j}}\left(x_{2}, u_{2}\right)$ given by (19) and (20), respectively.

Definition 9 (left-sided QLCTs). Let $A_{i}=\left[\begin{array}{cc}a_{i} & b_{i} \\ c_{i} & d_{i}\end{array}\right] \in \mathbb{R}^{2 \times 2}$ be a matrix parameter such that $\operatorname{det}\left(A_{i}\right)=1, b_{i} \neq 0$ for $i=$ 1,2 . The left-sided QLCTs of signals $f \in L^{1}\left(\mathbb{R}^{2} ; \mathbb{H}\right)$ are the functions $\mathscr{L}_{l}^{\mathbf{i}, \mathbf{j}}(f) \in L\left(\mathbb{R}^{2} ; \mathbb{W}\right)$ given by

$$
\begin{aligned}
& \mathscr{L}_{l}^{\mathbf{i}, \mathbf{j}}(f)\left(u_{1}, u_{2}\right) \\
& \quad:=\int_{\mathbb{R}^{2}} K_{A_{1}}^{\mathbf{i}}\left(x_{1}, u_{1}\right) K_{A_{2}}^{\mathbf{j}}\left(x_{2}, u_{2}\right) f\left(x_{1}, x_{2}\right) d x_{1} d x_{2},
\end{aligned}
$$

where the kernels $K_{A_{1}}^{\mathbf{i}}$ and $K_{A_{2}}^{\mathbf{j}}$ are given by (19) and (20), respectively.

Due to the validity of the Plancherel theorem, we study the right-sided QLCTs of 2D quaternionic signals in this paper.

Definition 10 (right-sided QLCTs). Let $A_{i}=\left[\begin{array}{ll}a_{i} & b_{i} \\ c_{i} & d_{i}\end{array}\right] \in \mathbb{R}^{2 \times 2}$ be a matrix parameter such that $\operatorname{det}\left(A_{i}\right)=1, b_{i} \neq 0$ for $i=$ 1,2 . The left-sided QLCTs of signals $f \in L^{1}\left(\mathbb{R}^{2} ; \mathbb{H}\right)$ are the functions $\mathscr{L}_{r}^{\mathbf{i}, \mathbf{j}}(f) \in L^{1}\left(\mathbb{R}^{2} ; \mathbb{W}\right)$ given by

$$
\begin{aligned}
& \mathscr{L}_{r}^{\mathbf{i}, \mathbf{j}}(f)\left(u_{1}, u_{2}\right) \\
& \quad:=\int_{\mathbb{R}^{2}} f\left(x_{1}, x_{2}\right) K_{A_{1}}^{\mathbf{i}}\left(x_{1}, u_{1}\right) K_{A_{2}}^{\mathbf{j}}\left(x_{2}, u_{2}\right) d x_{1} d x_{2},
\end{aligned}
$$

where $K_{A_{1}}^{\mathbf{i}}$ and $K_{A_{2}}^{\mathbf{j}}$ are given by (19) and (20), respectively.

It is significant to note that when $A_{1}=A_{2}=\left[\begin{array}{cc}0 & 1 \\ -1 & 0\end{array}\right]$, the QLCT of $f$ reduces to the QFT of $f$. We denote it by

$$
\underset{r}{\mathscr{F}_{r}^{\mathbf{i}, \mathbf{j}}}(f)\left(u_{1}, u_{2}\right):=\int_{\mathbb{R}^{2}} f\left(x_{1}, x_{2}\right) e^{-\mathbf{i} x_{1} u_{1}} e^{-\mathbf{j} x_{2} u_{2}} d x_{1} d x_{2} .
$$


Remark 11. In fact, the right-sided QLCTs defined above can be generalized as follows:

$$
\begin{aligned}
& \mathscr{L}_{r}^{\mathbf{e}_{1}, \mathbf{e}_{2}}(f)\left(u_{1}, u_{2}\right) \\
& \quad=\int_{\mathbb{R}^{2}} f\left(x_{1}, x_{2}\right) K_{A_{1}}^{\mathbf{e}_{1}}\left(x_{1}, u_{1}\right) K_{A_{2}}^{\mathbf{e}_{2}}\left(x_{2}, u_{2}\right) d x_{1} d x_{2},
\end{aligned}
$$

where $\mathbf{e}_{1}=\mathbf{e}_{1, \mathbf{i}} \mathbf{i}+\mathbf{e}_{1, \mathbf{j}} \mathbf{j}+\mathbf{e}_{1, \mathbf{k}} \mathbf{k}$ and $\mathbf{e}_{2}=\mathbf{e}_{2, \mathbf{i}} \mathbf{i}+\mathbf{e}_{2, \mathbf{j}} \mathbf{j}+\mathbf{e}_{2, \mathbf{k}} \mathbf{k}$ so that

$$
\begin{gathered}
\mathbf{e}_{1, \mathbf{i}}^{2}+\mathbf{e}_{1, \mathbf{j}}^{2}+\mathbf{e}_{1, \mathbf{k}}^{2}=\mathbf{e}_{2, \mathbf{i}}^{2}+\mathbf{e}_{2, \mathbf{j}}^{2}+\mathbf{e}_{2, \mathbf{k}}^{2}=1 \\
\left(\text { i.e., } \mathbf{e}_{1}^{2}=\mathbf{e}_{2}^{2}=-1\right) \\
\mathbf{e}_{1, \mathbf{i}} \mathbf{e}_{2, \mathbf{i}}+\mathbf{e}_{1, \mathbf{j}} \mathbf{e}_{2, \mathbf{j}}+\mathbf{e}_{1, \mathbf{k}} \mathbf{e}_{2, \mathbf{k}}=0 .
\end{gathered}
$$

Equation (45) is the special case of (47) in which $\mathbf{e}_{1}=\mathbf{i}$ and $\mathbf{e}_{2}=\mathbf{j}$.

Remark 12. For $b_{i} \neq 0(i=1,2)$ and $f \in L^{1}\left(\mathbb{R}^{2} ; \mathbb{R}\right)$, the (rightsided) QLCT of a 2D signal $f \in L^{1}\left(\mathbb{R}^{2} ; \mathbb{U}\right)$ in (45) has the closed-form representation:

$$
\begin{aligned}
\mathscr{L}_{r}^{\mathbf{i}, \mathbf{j}}(f)\left(\omega_{1}, \omega_{2}\right) \\
\quad:=\Phi_{0}\left(\omega_{1}, \omega_{2}\right)+\Phi_{1}\left(\omega_{1}, \omega_{2}\right) \\
\quad+\Phi_{2}\left(\omega_{1}, \omega_{2}\right)+\Phi_{3}\left(\omega_{1}, \omega_{2}\right),
\end{aligned}
$$

where we put the integrals

$$
\begin{gathered}
\Phi_{0}\left(\omega_{1}, \omega_{2}\right) \\
=\int_{\mathbb{R}^{2}} f\left(x_{1}, x_{2}\right) \frac{1}{2 \pi \sqrt{\mathbf{k} b_{1} b_{2}}} \\
\quad \times \cos \left(\frac{a_{1}}{2 b_{1}} x_{1}^{2}-\frac{1}{b_{1}} x_{1} \omega_{1}+\frac{d_{1}}{2 b_{1}} \omega_{1}^{2}\right) \\
\quad \times \cos \left(\frac{a_{2}}{2 b_{2}} x_{2}^{2}-\frac{1}{b_{2}} x_{2} \omega_{2}+\frac{d_{2}}{2 b_{2}} \omega_{2}^{2}\right) d x_{1} d x_{2}, \\
\Phi_{1}\left(\omega_{1}, \omega_{2}\right) \\
=\int_{\mathbb{R}^{2}} f\left(x_{1}, x_{2}\right) \frac{\sqrt{\mathbf{i}}}{2 \pi \sqrt{\mathbf{j} b_{1} b_{2}}} \\
\quad \times \sin \left(\frac{a_{1}}{2 b_{1}} x_{1}^{2}-\frac{1}{b_{1}} x_{1} \omega_{1}+\frac{d_{1}}{2 b_{1}} \omega_{1}^{2}\right) \\
\quad \times \cos \left(\frac{a_{2}}{2 b_{2}} x_{2}^{2}-\frac{1}{b_{2}} x_{2} \omega_{2}+\frac{d_{2}}{2 b_{2}} \omega_{2}^{2}\right) d x_{1} d x_{2},
\end{gathered}
$$

$$
\begin{aligned}
& \Phi_{2}\left(\omega_{1}, \omega_{2}\right) \\
& =\int_{\mathbb{R}^{2}} f\left(x_{1}, x_{2}\right) \frac{\sqrt{\mathbf{j}}}{2 \pi \sqrt{\mathbf{i} b_{1} b_{2}}} \\
& \quad \times \cos \left(\frac{a_{1}}{2 b_{1}} x_{1}^{2}-\frac{1}{b_{1}} x_{1} \omega_{1}+\frac{d_{1}}{2 b_{1}} \omega_{1}^{2}\right) \\
& \quad \times \sin \left(\frac{a_{2}}{2 b_{2}} x_{2}^{2}-\frac{1}{b_{2}} x_{2} \omega_{2}+\frac{d_{2}}{2 b_{2}} \omega_{2}^{2}\right) d x_{1} d x_{2}, \\
& \Phi_{3}\left(\omega_{1}, \omega_{2}\right) \\
& =\int_{\mathbb{R}^{2}} f\left(x_{1}, x_{2}\right) \frac{\sqrt{\mathbf{k}}}{2 \pi \sqrt{b_{1} b_{2}}} \\
& \quad \times \sin \left(\frac{a_{1}}{2 b_{1}} x_{1}^{2}-\frac{1}{b_{1}} x_{1} \omega_{1}+\frac{d_{1}}{2 b_{1}} \omega_{1}^{2}\right) \\
& \quad \times \sin \left(\frac{a_{2}}{2 b_{2}} x_{2}^{2}-\frac{1}{b_{2}} x_{2} \omega_{2}+\frac{d_{2}}{2 b_{2}} \omega_{2}^{2}\right) d x_{1} d x_{2} .
\end{aligned}
$$

These equations clearly show how the QLCTs separate real signals into four quaternion components, that is, the eveneven, odd-even, even-odds and odd-odd components of $f$.

Let us give an example to illustrate expression (45).

Example 13. Consider the quaternionic distribution signal, that is, the QLCT kernel of (45)

$$
f\left(x_{1}, x_{2}\right)=K_{A_{2}}^{-\mathbf{j}}\left(x_{2}, u_{0}\right) K_{A_{1}}^{-\mathbf{i}}\left(x_{1}, v_{0}\right) .
$$

It is easy to see that the QLCT of $f$ is a Dirac quaternionic function; that is,

$$
\mathscr{L}_{r}^{\mathbf{i} \mathbf{j}}(f)\left(u_{1}, u_{2}\right)=(2 \pi)^{2} \delta\left(u_{1}, u_{2}-\underline{t}\right), \quad \underline{t}=t_{1} \mathbf{e}_{1}+t_{2} \mathbf{e}_{2}
$$

4.2. Properties. This subsection describes important properties of the QLCTs that will be used to establish the uncertainty principles for the QLCTs.

We now establish a relation between the right-sided LCTs and the right-sided QLCTs of 2D quaternion-valued signals.

Lemma 14. Let $A_{i}=\left[\begin{array}{cc}a_{i} & b_{i} \\ c_{i} & d_{i}\end{array}\right] \in \mathbb{R}^{2 \times 2}$ be a matrix parameter such that $\operatorname{det}\left(A_{i}\right)=1, b_{i} \neq 0$ for $i=1,2$. For $f \in L^{1}\left(\mathbb{R}^{2} ; \mathbb{H}\right)$, one has

$$
\mathscr{L}_{r}^{\mathbf{i} \mathbf{j}}(f)\left(u_{1}, u_{2}\right) L_{r}^{\mathbf{j}}\left(L_{r}^{\mathbf{i}}(f)\right)\left(u_{1}, u_{2}\right) .
$$


Proof. By using the defnition of right-sided QLCTs (45),

$$
\begin{aligned}
\mathscr{L}_{r}^{\mathbf{i}, \mathbf{j}} & (f)\left(u_{1}, u_{2}\right) \\
& =\int_{\mathbb{R}^{2}} f\left(x_{1}, x_{2}\right) K_{A_{1}}^{\mathbf{i}}\left(x_{1}, u_{1}\right) K_{A_{2}}^{\mathbf{j}}\left(x_{2}, u_{2}\right) d x_{1} d x_{2} \\
& =\int_{\mathbb{R}} L_{r}^{\mathbf{i}}(f)\left(u_{1}, x_{2}\right) K_{A_{2}}^{\mathbf{j}}\left(x_{2}, u_{2}\right) d x_{2} \\
& =L_{r}^{\mathbf{j}}\left(L_{r}^{\mathbf{i}}(f)\right)\left(u_{1}, u_{2}\right) .
\end{aligned}
$$

We then establish the Plancherel theorems, specific to the right-sided QLCTs.

Theorem 15 (the Plancherel theorems of QLCTs). For $i=$ 1,2 , let $f_{i} \in \mathbb{S}$; the inner product (8) of two quaternionic module functions and their QLCTs is related by

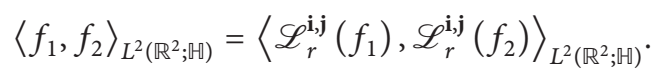

In particular, with $f_{1}=f_{2}=f$, we get the Parseval identity; that is,

$$
\|f\|_{L^{2}\left(\mathbb{R}^{2} ; \mathbb{H}\right)}^{2}=\left\|\mathscr{L}_{r}^{\mathbf{i}, \mathbf{j}}(f)\right\|_{L^{2}\left(\mathbb{R}^{2} ; \mathbb{\sharp}\right)}^{2} .
$$

Proof. By the inner product (8) and definition of right-sided QLCTs (45), a straightforward computation and Fubini's theorem show that

$$
\begin{aligned}
& \left\langle\mathscr{L}_{r}^{\mathbf{i}, \mathbf{j}}\left(f_{1}\right), \mathscr{L}_{r}^{\mathbf{i}, \mathbf{j}}\left(f_{2}\right)\right\rangle \\
& =\int_{\mathbb{R}^{2}}\left(\int_{\mathbb{R}^{2}} f_{1}\left(x_{1}, x_{2}\right) K_{A_{1}}^{\mathbf{i}}\left(x_{1}, u_{1}\right) K_{A_{2}}^{\mathbf{j}}\left(x_{2}, u_{2}\right) d x_{1} d x_{2}\right) \\
& \quad \times\left(\overline{\left.\int_{\mathbb{R}^{2}} f_{2}\left(y_{1}, y_{2}\right) K_{A_{1}}^{\mathbf{i}}\left(y_{1}, u_{1}\right) K_{A_{2}}^{\mathbf{j}}\left(y_{2}, u_{2}\right) d y_{1} d y_{2}\right)}\right) d u_{1} d u_{2} \\
& =\int_{\mathbb{R}^{6}} f_{1}\left(x_{1}, x_{2}\right) K_{A_{1}}^{\mathbf{i}}\left(x_{1}, u_{1}\right) \\
& \quad \times\left(K_{A_{2}}^{\mathbf{j}}\left(x_{2}, u_{2}\right) \overline{K_{A_{2}}^{\mathbf{j}}\left(y_{2}, u_{2}\right)}\right) \\
& =\int_{\mathbb{R}^{6}} \overline{f_{1}\left(y_{1}, y_{2}\right) K_{A_{1}}^{\mathbf{i}}\left(y_{1}, u_{1}\right)} d x_{1} d x_{2} d y_{1} d y_{2} d u_{1} d u_{2} \\
& \quad \times\left(\frac{1}{2 \pi b} e_{A_{1}}^{\mathbf{j}\left(a_{2} / 2 b_{2}\right)\left(x_{2}^{2}-y_{2}^{2}\right)} e^{-\mathbf{j}\left(1 / b_{2}\right) u_{2}\left(x_{2}-y_{2}\right)}\right) \\
& \quad \times \frac{f_{\mathbb{R}^{5}}\left(y_{1}, y_{2}\right) K_{A_{1}}^{\mathbf{i}}\left(y_{1}, u_{1}\right) d u_{1} d x_{1} d x_{2} d y_{1} d y_{2} d u_{2}}{f_{1}\left(x_{1}, x_{2}\right) K_{A_{1}}^{\mathbf{i}}\left(x_{1}, u_{1}\right)} \\
& \left.\quad \frac{f_{1}}{f_{1}}\right)
\end{aligned}
$$

$$
\begin{aligned}
& \times\left(e^{\mathbf{j}\left(a_{2} / 2 b_{2}\right)\left(x_{2}^{2}-y_{2}^{2}\right)} \delta\left(y_{2}-x_{2}\right)\right) \\
\times & \overline{f_{2}\left(y_{1}, y_{2}\right) K_{A_{1}}^{\mathbf{i}}\left(y_{1}, u_{1}\right)} d x_{1} d x_{2} d y_{1} d y_{2} d u_{2} \\
= & \left\langle L_{r}^{\mathbf{i}}\left(f_{1}\right), L_{r}^{\mathbf{i}}\left(f_{2}\right)\right\rangle=\left\langle f_{1}, f_{2}\right\rangle,
\end{aligned}
$$

where we have used the Plancherel theorem of right-sided LCTs (24) and formula (14).

Remark 16. Note that the Plancherel theorem is not valid for the two-sided or left-sided QLCT of quaternionic signals. For this reason, we choose to apply the right-sided QLCT of 2D quaternionic signals in the present paper.

Theorem 15 shows that the total signal energy computed in the spatial domain equals the total signal energy in the quaternionic domain. The Parseval theorem allows the energy of a quaternion-valued signal to be considered on either the spatial domain or the quaternionic domain and the change of domains for convenience of computation.

To proceed with, we prove the following derivative properties.

Lemma 17. For $i=1,2$, let $A_{i}=\left[\begin{array}{ll}a_{i} & b_{i} \\ c_{i} & d_{i}\end{array}\right] \in \mathbb{R}^{2 \times 2}$ be a matrix parameter, $b_{i} \neq 0$, and $a_{i} d_{i}-b_{i} c_{i}=1$. If $f \in \mathbb{S}$, then

$$
\begin{aligned}
\int_{\mathbb{R}^{2}} u_{i}^{2}\left|\mathscr{L}_{r}^{\mathbf{i}, \mathbf{j}}(f)\left(u_{1}, u_{2}\right)\right|^{2} d u_{1} d u_{2} \\
=b_{i}^{2} \int_{\mathbb{R}^{2}}\left|\frac{\partial}{\partial x_{i}} f\left(x_{1}, x_{2}\right)\right|^{2} d x_{1} d x_{2} .
\end{aligned}
$$

Proof. For $i=1$, using (14), (15), and Fubini's theorem, we have

$$
\begin{aligned}
& \int_{\mathbb{R}^{2}} u_{1}^{2}\left|\mathscr{L}_{r}^{\mathbf{i} \mathbf{j}}(f)\left(u_{1}, u_{2}\right)\right|^{2} d u_{1} d u_{2} \\
& =\int_{\mathbb{R}^{2}} u_{1}^{2}\left(\int_{\mathbb{R}^{2}} f\left(s_{1}, s_{2}\right) K_{A_{1}}^{\mathbf{i}}\left(s_{1}, u_{1}\right) K_{A_{2}}^{\mathbf{j}}\left(s_{2}, u_{2}\right) d s_{1} d s_{2}\right) \\
& \times\left(\overline{\int_{\mathbb{R}^{2}} f\left(x_{1}, x_{2}\right) K_{A_{1}}^{\mathbf{i}}\left(x_{1}, u_{1}\right) K_{A_{2}}^{\mathbf{j}}\left(x_{2}, u_{2}\right) d x_{1} d x_{2}}\right) d u_{1} d u_{2} \\
& =\int_{\mathbb{R}^{6}} u_{1}^{2} f\left(s_{1}, s_{2}\right) K_{A_{1}}^{\mathbf{i}}\left(s_{1}, u_{1}\right)\left(K_{A_{2}}^{\mathbf{j}}\left(s_{2}, u_{2}\right) \overline{K_{A_{2}}^{\mathbf{j}}\left(x_{2}, u_{2}\right)}\right) \\
& \times \overline{K_{A_{1}}^{\mathbf{i}}\left(x_{1}, u_{1}\right)} \overline{f\left(x_{1}, x_{2}\right)} d u_{2} d s_{1} d s_{2} d x_{1} d x_{2} d u_{1} \\
& =\int_{\mathbb{R}^{5}} u_{1}^{2} f\left(s_{1}, s_{2}\right) K_{A_{1}}^{\mathbf{i}}\left(s_{1}, u_{1}\right)\left(e^{\mathbf{j}\left(a_{2} / 2 b_{2}\right)\left(s_{2}^{2}-x_{2}^{2}\right)} \delta\left(x_{2}-s_{2}\right)\right) \\
& \times \overline{K_{A_{1}}^{\mathbf{i}}\left(x_{1}, u_{1}\right)} \overline{f\left(x_{1}, x_{2}\right)} d s_{1} d s_{2} d x_{1} d x_{2} d u_{1} \\
& =\int_{\mathbb{R}^{4}} f\left(s_{1}, s_{2}\right)\left(u_{1}^{2} K_{A_{1}}^{\mathbf{i}}\left(s_{1}, u_{1}\right) \overline{K_{A_{1}}^{\mathbf{i}}\left(x_{1}, u_{1}\right)}\right) \\
& \times \overline{f\left(x_{1}, x_{2}\right)} d u_{1} d s_{1} d x_{1} d x_{2}
\end{aligned}
$$




$$
\begin{aligned}
& =-b_{1}^{2} \int_{\mathbb{R}^{3}} f\left(s_{1}, x_{2}\right) \\
& \quad \times\left(e^{\mathbf{i}\left(a_{1} / 2 b_{1}\right)\left(s_{1}^{2}-x_{1}^{2}\right)} \frac{\partial^{2}}{\partial x_{1}^{2}} \delta\left(x_{1}-s_{1}\right)\right) \overline{f\left(x_{1}, x_{2}\right)} d s_{1} d x_{1} d x_{2} \\
& =-b_{1}^{2} \int_{\mathbb{R}^{2}} f\left(x_{1}, x_{2}\right) \frac{\partial^{2}}{\partial x_{1}^{2}} \overline{f\left(x_{1}, x_{2}\right)} d x_{1} d x_{2} \\
& =b_{1}^{2} \int_{\mathbb{R}^{2}}\left|\frac{\partial}{\partial x_{1}} f\left(x_{1}, x_{2}\right)\right|^{2} d x_{1} d x_{2} .
\end{aligned}
$$

To prove the case $i=2$, we argue in the same spirit as in the proof of the case $i=1$. Applying (14), (15), and Fubini's theorem, we have

$$
\begin{aligned}
& \int_{\mathbb{R}^{2}} u_{2}^{2}\left|\mathscr{L}_{r}^{\mathbf{i} \mathbf{j}}(f)\left(u_{1}, u_{2}\right)\right|^{2} d u_{1} d u_{2} \\
& =\int_{\mathbb{R}^{2}} u_{2}^{2}\left(\int_{\mathbb{R}^{2}} f\left(s_{1}, s_{2}\right) K_{A_{1}}^{\mathbf{i}}\left(s_{1}, u_{1}\right) K_{A_{2}}^{\mathbf{j}}\left(s_{2}, u_{2}\right) d s_{1} d s_{2}\right) \\
& \times\left(\overline{\int_{\mathbb{R}^{2}} f\left(x_{1}, x_{2}\right) K_{A_{1}}^{\mathbf{i}}\left(x_{1}, u_{1}\right) K_{A_{2}}^{\mathbf{j}}\left(x_{2}, u_{2}\right) d x_{1} d x_{2}}\right) d u_{1} d u_{2} \\
& =\int_{\mathbb{R}^{6}} f\left(s_{1}, s_{2}\right) K_{A_{1}}^{\mathbf{i}}\left(s_{1}, u_{1}\right)\left(u_{2}^{2} K_{A_{2}}^{\mathbf{j}}\left(s_{2}, u_{2}\right) \overline{K_{A_{2}}^{\mathbf{j}}\left(x_{2}, u_{2}\right)}\right) \\
& \times \overline{f\left(x_{1}, x_{2}\right) K_{A_{1}}^{\mathbf{i}}\left(x_{1}, u_{1}\right)} d u_{2} d s_{1} d s_{2} d x_{1} d x_{2} d u_{1} \\
& =-b_{2}^{2} \int_{\mathbb{R}^{5}} f\left(s_{1}, s_{2}\right) K_{A_{1}}^{\mathbf{i}}\left(s_{1}, u_{1}\right) \\
& \times\left(e^{\mathbf{j}\left(a_{2} / 2 b_{2}\right)\left(s_{2}^{2}-x_{2}^{2}\right)} \frac{\partial^{2}}{\partial x_{2}^{2}} \delta\left(x_{2}-s_{2}\right)\right) \\
& \times \overline{f\left(x_{1}, x_{2}\right) K_{A_{1}}^{\mathbf{i}}\left(x_{1}, u_{1}\right)} d s_{1} d s_{2} d x_{1} d x_{2} d u_{1} \\
& =-b_{2}^{2} \int_{\mathbb{R}^{5}} f\left(s_{1}, x_{2}\right) K_{A_{1}}^{\mathbf{i}}\left(s_{1}, u_{1}\right) \\
& \times \overline{\left(\frac{\partial^{2}}{\partial x_{2}^{2}} f\left(x_{1}, x_{2}\right)\right) K_{A_{1}}^{\mathbf{i}}\left(x_{1}, u_{1}\right)} d s_{1} d x_{1} d x_{2} d u_{1} \\
& =-b_{2}^{2} \int_{\mathbb{R}^{4}} f\left(s_{1}, x_{2}\right)\left(K_{A_{1}}^{\mathbf{i}}\left(s_{1}, u_{1}\right) \overline{K_{A_{1}}^{\mathbf{i}}\left(x_{1}, u_{1}\right)}\right) \\
& \times \overline{\frac{\partial^{2}}{\partial x_{2}^{2}} f\left(x_{1}, x_{2}\right)} d u_{1} d s_{1} d x_{1} d x_{2} \\
& =-b_{2}^{2} \int_{\mathbb{R}^{3}} f\left(s_{1}, x_{2}\right)\left(e^{\mathbf{i}\left(a_{1} / 2 b_{1}\right)\left(s_{1}^{2}-x_{1}^{2}\right)} \delta\left(x_{1}-s_{1}\right)\right) \\
& \times \overline{\frac{\partial^{2}}{\partial x_{2}^{2}} f\left(x_{1}, x_{2}\right)} d s_{1} d x_{1} d x_{2} \\
& =-b_{2}^{2} \int_{\mathbb{R}^{2}} f\left(x_{1}, x_{2}\right) \frac{\partial^{2}}{\partial x_{2}^{2}} \overline{f\left(x_{1}, x_{2}\right)} d x_{1} d x_{2} \\
& =b_{2}^{2} \int_{\mathbb{R}^{2}}\left|\frac{\partial}{\partial x_{2}} f\left(x_{1}, x_{2}\right)\right|^{2} d x_{1} d x_{2} \text {. }
\end{aligned}
$$

Some properties of the QLCT are summarized in Table 1. Let $f_{1}$ and $f_{2} \in \mathbb{S}$, the constants $\alpha$ and $\beta \in \mathbb{R}, A_{i}=\left[\begin{array}{cc}a_{i} & b_{i} \\ c_{i} & d_{i}\end{array}\right] \in$ $\mathbb{R}^{2 \times 2}, b_{i} \neq 0$, and $a_{i} d_{i}-b_{i} c_{i}=1$.

\section{Uncertainty Principles for QLCTs}

In signal processing much effort has been placed in the study of the classical Heisenberg uncertainty principle during the last years. Shinde and Gadre [9] established an uncertainty principle for fractional Fourier transforms that provides a lower bound on the uncertainty product of real signal representations in both time and frequency domains. Korn [50] proposed Heisenberg-type uncertainty principles for Cohen transforms which describe lower limits for the time frequency concentration. In the meantime, Hitzer et al. [51-54] investigated a directional uncertainty principle for the CliffordFourier transform, which describes how the variances (in arbitrary but fixed directions) of a multivector-valued function and its Clifford-Fourier transform are related. On our knowledge, a systematic work on the investigation of uncertainty relations using the QLCT of a multivector-valued function has not been carried out.

In the following we explicitly prove and generalize the classical uncertainty principle to quaternionic module functions using the QLCTs. We also give an explicit proof for the Gaussian quaternionic functions (the Gabor filters) to be indeed the only functions that minimize the uncertainty. We further emphasize that our generalization is nontrivial because the multiplication of quaternions and the quaternionic linear canonical kernel are both noncommutative. For this purpose we introduce the following definition.

Definition 18. For $k=1,2$, let $f, x_{k} f \in L^{2}\left(\mathbb{R}^{2} ; \mathbb{H}\right)$ and $\mathscr{L}_{r}^{\mathbf{i}, \mathbf{j}}(f)$, $u_{k} \mathscr{L}_{r}^{\mathbf{i}, \mathbf{j}}(f) \in L^{2}\left(\mathbb{R}^{2} ; \mathbb{H}\right)$. Then the effective spatial width or spatial uncertainty $\Delta x_{k}$ of $f$ is evaluated by

$$
\Delta x_{k}:=\sqrt{\operatorname{Var}_{k}(f)}
$$

where $\operatorname{Var}_{k}(f)$ is the variance of the energy distribution of $f$ along the $x_{k}$-axis defined by

$$
\begin{aligned}
\operatorname{Var}_{k}(f) & :=\frac{\left\|x_{k} f\right\|_{L^{2}\left(\mathbb{R}^{2} ; \mathbb{\sharp}\right)}^{2}}{\|f\|_{L^{2}\left(\mathbb{R}^{2} ; \mathbb{}\right)}^{2}} \\
& =\frac{\int_{\mathbb{R}^{2}} x_{k}^{2}\left|f\left(x_{1}, x_{2}\right)\right|^{2} d x_{1} d x_{2}}{\int_{\mathbb{R}^{2}}\left|f\left(x_{1}, x_{2}\right)\right|^{2} d x_{1} d x_{2}} .
\end{aligned}
$$

Similarly, in the quaternionic domain we define the effective spectral width as

$$
\Delta u_{k}:=\sqrt{\operatorname{Var}_{k}\left(\mathscr{L}_{r}^{\mathbf{i}, \mathbf{j}}(f)\right)}
$$


TABLE 1: Properties of the QLCT.

\begin{tabular}{lcc}
\hline Property & Function & QLCT \\
\hline $\begin{array}{l}\text { Real linearity } \\
\text { Formula } \\
\text { Plancherel } \\
\text { Parseval }\end{array}$ & $\alpha f_{1}\left(x_{1}, x_{2}\right)+\beta f_{2}\left(x_{1}, x_{2}\right)$ & $\alpha \mathscr{L}_{r}^{\mathbf{i}, \mathbf{j}}(f)\left(u_{1}, u_{2}\right)+\beta \mathscr{L}_{r}^{\mathbf{i}, \mathbf{j}}\left(f_{2}\right)\left(u_{1}, u_{2}\right)$ \\
Derivatives & $\left\langle f_{1}, f_{2}\right\rangle_{L^{2}\left(\mathbb{R}^{2} ; \mathbb{H}\right)}$ & $\left\langle\mathscr{L}_{r}^{\mathbf{i}, \mathbf{j}}\left(f_{1}\right), \mathscr{L}_{r}^{\mathbf{i}, \mathbf{j}}\left(f_{2}\right)\right\rangle_{L^{2}\left(\mathbb{R}^{2} ; \mathbb{H}\right)}$ \\
\hline
\end{tabular}

where $\operatorname{Var}_{k}\left(\mathscr{L}_{r}^{\mathbf{i}, \mathbf{j}}(f)\right)$ is the variance of the frequency spectrum of $f$ along the $u_{k}$ frequency axis given by

$$
\begin{aligned}
& \operatorname{Var}_{k}\left(\mathscr{L}_{r}^{\mathbf{i}, \mathbf{j}}(f)\right)
\end{aligned}
$$

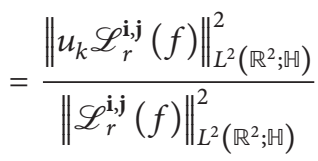

$$
\begin{aligned}
& =\frac{\int_{\mathbb{R}^{2}} u_{k}^{2}\left|\mathscr{L}_{r}^{\mathbf{i}, \mathbf{j}}(f)\left(u_{1}, u_{2}\right)\right|^{2} d u_{1} d u_{2}}{\int_{\mathbb{R}^{2}}\left|\mathscr{L}_{r}^{\mathbf{i}, \mathbf{j}}(f)\left(u_{1}, u_{2}\right)\right|^{2} d u_{1} d u_{2}} .
\end{aligned}
$$

Example 19. Let us consider a 2D Gaussian quaternionic function (Figures 1, 2, 3, and 4) of the form

$$
f\left(x_{1}, x_{2}\right)=C e^{-\left(\alpha_{1} x_{1}^{2}+\alpha_{2} x_{2}^{2}\right)},
$$

where $C=C_{i 0}+\mathbf{i} C_{i 1}+\mathbf{j} C_{i 2}+\mathbf{k} C_{i 3} \in \mathbb{H}$, for $i=1,2$, are quaternionic constants and $\alpha_{1}, \alpha_{2} \in \mathbb{R}$ are positive real constants.

Then the QLCT of $f$ is given by

$$
\begin{aligned}
& \mathscr{L}^{\mathbf{i}, \mathbf{j}}(f)\left(u_{1}, u_{2}\right) \\
&= C\left(\int_{\mathbb{R}} K_{A_{1}}^{\mathbf{i}}\left(x_{1}, u_{1}\right) e^{-\alpha_{1} x_{1}^{2}} d x_{1}\right) \\
& \times\left(\int_{\mathbb{R}} e^{-\alpha_{2} x_{2}^{2}} K_{A_{2}}^{\mathbf{j}}\left(x_{2}, u_{2}\right) d x_{2}\right) \\
&= C \sqrt{\frac{2 b_{1} \pi}{2 \alpha_{1} b_{1}-a_{1} \mathbf{i}}} \\
& \times e^{\left(\left(\left(4 \alpha_{1} b_{1}-2 a_{1} \mathbf{i}\right) d_{1}+2\right) /\left(2 b_{1}\left(4 \alpha_{1} b_{1}-2 a_{1} \mathbf{i}\right)\right)\right) u_{1}^{2}} \\
& \times e^{\left(\left(\left(4 \alpha_{2} b_{2}-2 a_{2} \mathbf{j}\right) d_{2}+2\right) /\left(2 b_{2}\left(4 \alpha_{2} b_{2}-2 a_{2} \mathbf{j}\right)\right)\right) u_{2}^{2}} \\
& \times \sqrt{\frac{2 b_{2} \pi}{2 \alpha_{2} b_{2}-a_{2} \mathbf{j}}} .
\end{aligned}
$$

This shows that the QLCT of the Gaussian quaternionic function is another Gaussian quaternionic function.

Figures 1 and 2 visualize the quaternionic Gaussian function, for $\alpha_{1}=\alpha_{2}=3$, and $\alpha_{1}=3$ and $\alpha_{2}=1$ in the spatial domain. Figures 3 and 4 visualize the quaternionic Gaussian function, for $\alpha_{1}=1$ and $\alpha_{2}=3$, and $\alpha_{1}=\alpha_{2}=1 / 2$ in the spatial domain.
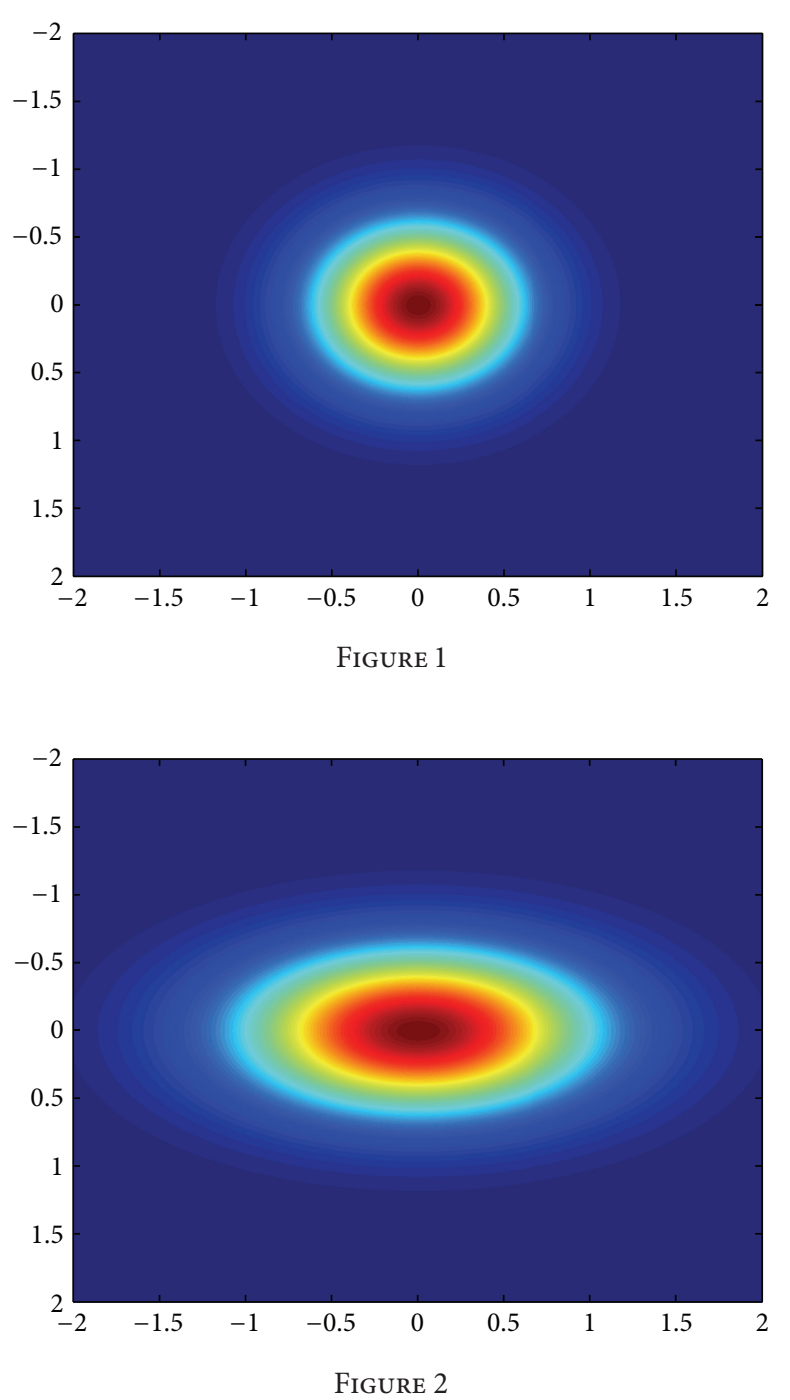

Now let us begin the proofs of two uncertainty relations.

Theorem 20. For $k=1,2$, let $f \in \mathbb{S}$. Then the following uncertainty relations are fulfilled:

$$
\begin{aligned}
& \Delta x_{1} \Delta u_{1} \geq \frac{b_{1}}{2}, \\
& \Delta x_{2} \Delta u_{2} \geq \frac{b_{2}}{2} .
\end{aligned}
$$




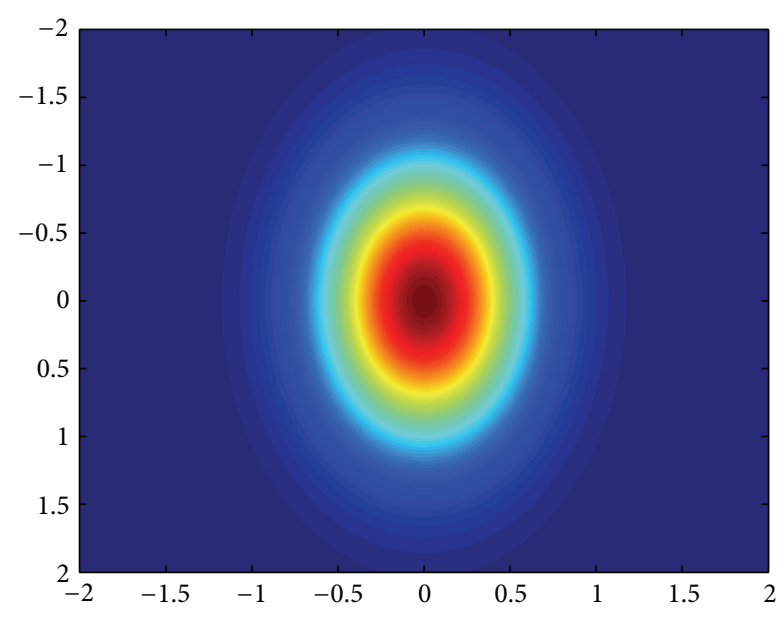

FIGURE 3

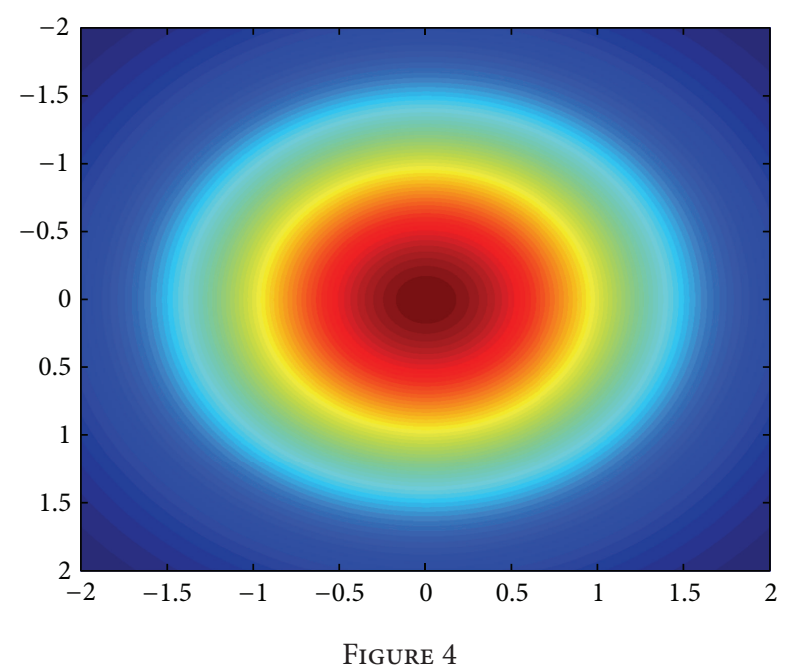

The combination of the two spatial uncertainty principles above leads to the uncertainty principle for the $2 \mathrm{D}$ quaternionic signal $f\left(x_{1}, x_{2}\right)$ of the form

$$
\Delta x_{1} \Delta x_{2} \Delta u_{1} \Delta u_{2} \geq \frac{b_{1} b_{2}}{4}
$$

Equality holds in (68) if and only if $f$ is a 2D Gaussian function; that is.

$$
f\left(x_{1}, x_{2}\right)=\beta e^{-\left(C_{1} x_{1}^{2}+C_{2} x_{2}^{2}\right) / 2},
$$

where $C_{1}, C_{2}$ are positive real constants and $\beta=$ $\|f\|_{L^{2}\left(\mathbb{R}^{2} ; \uplus\right)}\left(C_{1} C_{2} / \pi^{2}\right)^{1 / 4}$.
Proof. Applying (58) in Lemma 17 and using the Schwarz inequality (10), we have

$$
\begin{aligned}
& \left(\int_{\mathbb{R}^{2}} x_{k}^{2}\left|f\left(x_{1}, x_{2}\right)\right|^{2} d x_{1} d x_{2}\right) \\
& \quad \times\left(\int_{\mathbb{R}^{2}} u_{k}^{2}\left|\mathscr{L}_{r}^{\mathbf{i}, \mathbf{j}}(f)\left(u_{1}, u_{2}\right)\right|^{2} d u_{1} d u_{2}\right) \\
& =\left(\int_{\mathbb{R}^{2}} x_{k}^{2}\left|f\left(x_{1}, x_{2}\right)\right|^{2} d x_{1} d x_{2}\right) \\
& \quad \times\left(b_{k}^{2} \int_{\mathbb{R}^{2}}\left|\frac{\partial}{\partial x_{k}} f\left(x_{1}, x_{2}\right)\right|^{2} d x_{1} d x_{2}\right) \\
& \geq b_{k}^{2}\left|\int_{\mathbb{R}^{2}} x_{k} \frac{\partial}{f\left(x_{1}, x_{2}\right)} \frac{\partial}{\partial x_{k}} f\left(x_{1}, x_{2}\right) d x_{1} d x_{2}\right|^{2} .
\end{aligned}
$$

Using the exponential form of a 2D quaternionic signal (6), let

$$
f\left(x_{1}, x_{2}\right)=f_{0}\left(x_{1}, x_{2}\right)+\underline{f}\left(x_{1}, x_{2}\right)=\left|f\left(x_{1}, x_{2}\right)\right| e^{\underline{\underline{e}} \alpha},
$$

where $\underline{e}=f\left(x_{1}, x_{2}\right) /\left|f\left(x_{1}, x_{2}\right)\right|$ and $\alpha=\arctan \left(\left|f\left(x_{1}, x_{2}\right)\right| /\right.$ $\left.f_{0}\left(x_{1}, x_{2}\right)\right)$; then

$$
\begin{aligned}
x_{k} & \overline{f\left(x_{1}, x_{2}\right)} \frac{\partial}{\partial x_{k}} f\left(x_{1}, x_{2}\right) \\
= & x_{k}\left|f\left(x_{1}, x_{2}\right)\right| e^{-\underline{e} \alpha} \frac{\partial}{\partial x_{k}}\left(\left|f\left(x_{1}, x_{2}\right)\right| e^{\underline{e} \alpha}\right) \\
= & x_{k}\left|f\left(x_{1}, x_{2}\right)\right| e^{-\underline{e} \alpha} \\
& \times \\
= & \left.\quad x_{k}\left|f\left(\frac{\partial}{\partial x_{k}}\left|f\left(x_{1}, x_{2}\right)\right|\right) e^{\underline{e} \alpha}+\right| f\left(x_{1}, x_{2}\right) \mid\left(\frac{\partial}{\partial x_{k}} e^{\underline{e} \alpha}\right)\right] \\
& +x_{k}\left|f\left(x_{1}, x_{2}\right)\right|^{2}\left(\frac{\partial}{\partial x_{k}}(\underline{e} \alpha)\right) \\
= & \frac{1}{2} \frac{\partial}{\partial x_{k}}\left(x_{k}\left|f\left(x_{1}, x_{2}\right)\right|^{2}\right)-\frac{1}{2}\left|f\left(x_{1}, x_{2}\right)\right|^{2} \\
& +x_{k}\left|f\left(x_{1}, x_{2}\right)\right|^{2}\left(\frac{\partial}{\partial x_{k}}(\underline{e} \alpha)\right) .
\end{aligned}
$$

Therefore,

$$
\begin{gathered}
b_{k}^{2}\left|\int_{\mathbb{R}^{2}} x_{k} \overline{f\left(x_{1}, x_{2}\right)} \frac{\partial}{\partial x_{k}} f\left(x_{1}, x_{2}\right) d x_{1} d x_{2}\right|^{2} \\
=\left.b_{k}^{2}\left|\int_{\mathbb{R}^{2}} \frac{1}{2} \frac{\partial}{\partial x_{k}}\left(x_{k}\left|f\left(x_{1}, x_{2}\right)\right|^{2}\right)-\frac{1}{2}\right| f\left(x_{1}, x_{2}\right)\right|^{2} \\
+\left.x_{k}\left|f\left(x_{1}, x_{2}\right)\right|^{2}\left(\frac{\partial}{\partial x_{k}}(\underline{e} \alpha)\right) d x_{1} d x_{2}\right|^{2}
\end{gathered}
$$

The first term is a perfect differential and integrates to zero. The second term gives minus one half of the energy $\|f\|_{L^{2}\left(\mathbb{R}^{2} ; \mathbb{W}\right)}^{2}$. 
Hence

$$
\begin{aligned}
& \left(\int_{\mathbb{R}^{2}} x_{k}^{2}\left|f\left(x_{1}, x_{2}\right)\right|^{2} d x_{1} d x_{2}\right) \\
& \quad \times\left(\int_{\mathbb{R}^{2}} u_{k}^{2}\left|\mathscr{L}_{r}^{\mathbf{i}, \mathbf{j}}(f)\left(u_{1}, u_{2}\right)\right|^{2} d u_{1} d u_{2}\right) \\
& \geq b_{k}^{2}\left|-\frac{1}{2}\|f\|_{L^{2}\left(\mathbb{R}^{2} ; \mathbb{*}\right)}^{2}\right|^{2}=\frac{b_{k}^{2}}{4}\|f\|_{L^{2}\left(\mathbb{R}^{2} ; \mathbb{U}\right)}^{4} .
\end{aligned}
$$

By definitions of $\Delta x_{k}, \Delta u_{k}$, and Parseval theorem (56), we have

$$
\begin{aligned}
\left(\Delta x_{k} \Delta u_{k}\right)^{2} & \left(\left(\int_{\mathbb{R}^{2}} x_{k}^{2}\left|f\left(x_{1}, x_{2}\right)\right|^{2} d x_{1} d x_{2}\right)\right. \\
& \left.\times\left(\int_{\mathbb{R}^{2}} u_{k}^{2}\left|\mathscr{L}_{r}^{\mathbf{i}, \mathbf{j}}(f)\left(u_{1}, u_{2}\right)\right|^{2} d u_{1} d u_{2}\right)\right) \\
& \times\left(\left(\int_{\mathbb{R}^{2}}\left|f\left(x_{1}, x_{2}\right)\right|^{2} d x_{1} d x_{2}\right)\right. \\
& \left.\times\left(\int_{\mathbb{R}^{2}}\left|\mathscr{L}_{r}^{\mathbf{i} \mathbf{j}}(f)\left(u_{1}, u_{2}\right)\right|^{2} d u_{1} d u_{2}\right)\right)^{-1} \\
= & \left(\left(\int_{\mathbb{R}^{2}} x_{k}^{2}\left|f\left(x_{1}, x_{2}\right)\right|^{2} d x_{1} d x_{2}\right)\right. \\
& \left.\times\left(\int_{\mathbb{R}^{2}} u_{k}^{2}\left|\mathscr{L}_{r}^{\mathbf{i}, \mathbf{j}}(f)\left(u_{1}, u_{2}\right)\right|^{2} d u_{1} d u_{2}\right)\right) \\
\quad & \left(\|f\|_{L^{2}\left(\mathbb{R}^{2} ; \boxplus\right)}^{4}\right)^{-1} \\
\geq & \frac{b_{k}^{2}}{4}
\end{aligned}
$$

and therefore we have the uncertainty principle as given by (67) and (68).

We finally show that the equality in (67) and (68) is satisfied if and only if $f$ is a Gaussian quaternionic function.

Since the minimum value for the uncertainty product is $b_{k} / 2$, we can ask what signals have that minimum value. The Schwarz inequality (10) becomes an equality when the two functions are proportional to each other. Hence, we take $g=$ $-C f$, where $C$ is a quaternionic constant and the -1 has been inserted for convenience. We therefore have

$$
\frac{\partial}{\partial x_{k}} f\left(x_{1}, x_{2}\right)=-C_{k} x_{k} f\left(x_{1}, x_{2}\right) \text {. }
$$

This is a necessary condition for the uncertainty product to be the minimum. But it is not sufficient since we must also have the term

$$
\int_{\mathbb{R}^{2}} x_{k}\left|f\left(x_{1}, x_{2}\right)\right|^{2}\left(\frac{\partial}{\partial x_{k}}(\underline{e} \alpha)\right) d x_{1} d x_{2}=0
$$

because by (73), we see that is the only way we can actually get the value of $b_{k} / 2$.
Since $C_{k}$ is arbitrary we can write it in terms of its scalar and nonscalar parts, $C_{k}:=\operatorname{Sc}\left(C_{k}\right)+\operatorname{NSc}\left(C_{k}\right)$. The solution of (76) is hence

$$
\begin{aligned}
f\left(x_{1}, x_{2}\right)= & \beta e^{-\left(C_{1} x_{1}^{2}+C_{2} x_{2}^{2}\right) / 2} \\
= & \beta e^{-\left(\operatorname{Sc}\left(C_{1}\right) x_{1}^{2}+\operatorname{Sc}\left(C_{2}\right) x_{2}^{2}\right) / 2} \\
& \times e^{-\left(\operatorname{NSc}\left(C_{1}\right) x_{1}^{2}+\operatorname{NSc}\left(C_{2}\right) x_{2}^{2}\right) / 2},
\end{aligned}
$$

for some constant $\beta$. Since $\underline{e} \alpha=-\left(\operatorname{NSc}\left(C_{1}\right) x_{1}^{2}+\operatorname{NSc}\left(C_{2}\right) x_{2}^{2}\right) /$ 2 , it follows that

$$
\frac{\partial}{\partial x_{k}}(\underline{e} \alpha)=-\operatorname{NSc}\left(C_{k}\right) x_{k}
$$

We have

$$
\begin{aligned}
\int_{\mathbb{R}^{2}} & x_{k}\left|f\left(x_{1}, x_{2}\right)\right|^{2}\left(\frac{\partial}{\partial x_{k}}(\underline{e} \alpha)\right) d x_{1} d x_{2} \\
= & -\operatorname{NSc}\left(C_{k}\right) \beta^{2} \\
& \times \int_{\mathbb{R}^{2}} x_{k}^{2} e^{-\left(\operatorname{Sc}\left(C_{1}\right) x_{1}^{2}+\operatorname{Sc}\left(C_{2}\right) x_{2}^{2}\right)} d x_{1} d x_{2} .
\end{aligned}
$$

The only way this can be zero is if $\operatorname{NSc}\left(C_{k}\right)=0$ and hence $C_{k}$ must be a real number. We then have

$$
f\left(x_{1}, x_{2}\right)=\beta e^{-\left(C_{1} x_{1}^{2}+C_{2} x_{2}^{2}\right) / 2},
$$

where $C_{1}, C_{2}$ are positive real constants since $f \in \mathbb{S}$ and we have included the appropriate normalization $\beta=$ $\|f\|_{L^{2}\left(\mathbb{R}^{2} ; \mathbb{H}\right)}\left(C_{1} C_{2} / \pi^{2}\right)^{1 / 4}$.

Since the 2D Gaussian function $f\left(x_{1}, x_{2}\right)$ of (81) achieves the minimum width-bandwidth product, it is theoretically a very good prototype waveform. One can therefore construct a basic waveform using spatially or frequency-scaled versions of $f\left(x_{1}, x_{2}\right)$ to provide multiscale spectral resolution. Such a wavelet basis construction derived from a Gaussian quaternionic function prototype waveform has been realized, for example, in the quaternionic wavelet transforms in [55]. The optimal space-frequency localization is also another reason why 2D Clifford-Gabor bandpass filters were suggested in [56].

\section{Conclusion}

In this paper we developed the definition of QLCT. The various properties of QLCT such as partial derivative, the Plancherel, and Parseval theorems are discussed. Using the well-known properties of the classical LCT, we established an uncertainty principle for the QLCT. This uncertainty principle states that the product of the variances of quaternionvalued signals in the spatial and frequency domains has a lower bound. It is shown that only a $2 \mathrm{D}$ Gaussian signal minimizes the uncertainty. With the help of this principle, we hope to contribute to the theory and applications of signal processing through this investigation and to develop 
further general numerical methods for differential equations. The results in this paper are new in the literature. Further investigations on this topic are now under investigation and will be reported in a forthcoming paper.

\section{Acknowledgments}

The first author acknowledges financial support from the research Grant of the University of Macau no. MYRG142(Y1L2)-FST11-KKI and the Science and Technology Development Fund FDCT/094/2011A. This work was supported by FEDER funds through COMPETE-Operational Programme Factors of Competitiveness (Programa Operacional Factores de Competitividade) and by Portuguese funds through the Center for Research and Development in Mathematics and Applications (University of Aveiro) and the Portuguese Foundation for Science and Technology ("FCT-Fundação para a Ciência e a Tecnologia"), within project PEst-C/ MAT/UI4106/2011 with COMPETE no. FCOMP-01-0124FEDER-022690. Partial support from the Foundation for Science and Technology (FCT) via the postDoctoral grant SFRH/BPD/66342/2009 is also acknowledged by the third author.

\section{References}

[1] Z. X. Da, Modern Signal Processing, Tsinghua University Press, Beijing, China, 2nd edition, 2002.

[2] A. Dembo, T. M. Cover, and J. A. Thomas, "Informationtheoretic inequalities," IEEE Transactions on Information Theory, vol. 37, no. 6, pp. 1501-1518, 1991.

[3] G. Hardy, J. E. Littlewood, and G. Polya, Inequalities, Cambridge University Press, Cambridge, UK, 2nd edition, 1951.

[4] H. P. Heinig and M. Smith, "Extensions of the Heisenberg-Weyl inequality," International Journal of Mathematics and Mathematical Sciences, vol. 9, no. 1, pp. 185-192, 1986.

[5] P. J. Loughlin and L. Cohen, "The uncertainty principle: global, local, or both?" IEEE Transactions on Signal Processing, vol. 52, no. 5, pp. 1218-1227, 2004.

[6] D. Mustard, "Uncertainty principles invariant under the fractional Fourier transform," Australian Mathematical Society Journal B, vol. 33, no. 2, pp. 180-191, 1991.

[7] V. Majernik, M. Eva, and S. Shpyrko, "Uncertainty relations expressed by Shannon-like entropies," Central European Journal of Physics, vol. 3, pp. 393-420, 2003.

[8] H. M. Ozaktas and O. Aytür, "Fractional Fourier domains," Signal Processing, vol. 46, no. 1, pp. 119-124, 1995.

[9] S. Shinde and V. M. Gadre, "An uncertainty principle for real signals in the fractional Fourier transform domain," IEEE Transactions on Signal Processing, vol. 49, no. 11, pp. 2545-2548, 2001.

[10] A. Stern, "Sampling of compact signals in offset linear canonical transform domains," Signal, Image and Video Processing, vol. 1, no. 4, pp. 359-367, 2007.

[11] X. Guanlei, W. Xiaotong, and X. Xiaogang, "Generalized Hilbert transform and its properties in 2D LCT domain," Signal Processing, vol. 89, no. 7, pp. 1395-1402, 2009.

[12] O. Aytür and H. M. Ozaktas, "Non-orthogonal domains in phase space of quantum optics and their relation to fractional Fourier transforms," Optics Communications, vol. 120, no. 3-4, pp. 166-170, 1995.
[13] L. Cohen, "Uncertainty principles of windowed wave functions," Optics Communications, vol. 179, no. 1, pp. 221-229, 2000.

[14] B. B. Iwo, "Entropic uncertainty relations in quantum mechanics," in Quantum Probability and Applications II, L. Accardi and W. von Waldenfels, Eds., vol. 1136 of Lecture Notes in Mathematics, pp. 90-103, Springer, Berlin, Germany, 1985.

[15] B. B. Iwo, "Formulation of the uncertainty relations in terms of the Rényi entropies," Physical Review A, vol. 74, no. 5, Article ID 052101, 6 pages, 2006.

[16] B. B. Iwo, "Rényi entropy and the uncertainty relations," in Foundations of Probability and Physics, G. Adenier, C. A. Fuchs, A. Yu, and A. Khrennikov, Eds., vol. 889 of AIP Conference Proceedings, pp. 52-61, American Institute of Physics, Melville, NY, USA, 2007.

[17] H. Maassen and J. B. M. Uffink, "Generalized entropic uncertainty relations," Physical Review Letters, vol. 60, no. 12, pp. 11031106, 1988.

[18] H. Maassen, "A discrete entropic uncertainty relation," in Quantum Probability and Applications, vol. 1442 of Lecture Notes in Mathematics, pp. 263-266, Springer, Berlin, Germany, 1990.

[19] A. Renyi, "On measures of information and entropy," in Proccedinys of the 4th Berkeley Symposium on Mathematical Statistics and Probability, pp. 547-561, 1960.

[20] A. Stern, "Uncertainty principles in linear canonical transform domains and some of their implications in optics," Journal of the Optical Society of America A, vol. 25, no. 3, pp. 647-652, 2008.

[21] K. Wódkiewicz, "Operational approach to phase-space measurements in quantum mechanics," Physical Review Letters, vol. 52, no. 13, pp. 1064-1067, 1984.

[22] M. Bahri, E. S. M. Hitzer, A. Hayashi, and R. Ashino, "An uncertainty principle for quaternion Fourier transform," Computers \& Mathematics with Applications, vol. 56, no. 9, pp. 2398-2410, 2008.

[23] E. M. S. Hitzer, "Directional uncertainty principle for quaternion Fourier transform," Advances in Applied Clifford Algebras, vol. 20, no. 2, pp. 271-284, 2010.

[24] K. E. Nicewarner and A. C. Sanderson, "General representation for orientational uncertainty using random unit quaternions," in Proceedings of the 1994 IEEE International Conference on Robotics and Automation, pp. 1161-1168, May 1994.

[25] S. J. Sangwine and T. A. Ell, "Hypercomplex Fourier transforms of color images," IEEE Transactions on Image Processing, vol. 16, no. 1, pp. 22-35, 2007.

[26] P. Bas, N. le Bihan, and J. M. Chassery, "Color image watermarking using quaternion Fourier transform," in Proccedings of the IEEE International Conference on Accoustics, Speech, and Signal Processing (ICASSP '03), pp. 521-524, Hong-Kong, April 2003.

[27] E. Bayro-Corrochano, N. Trujillo, and M. Naranjo, "Quaternion Fourier descriptors for the preprocessing and recognition of spoken words using images of spatiotemporal representations," Journal of Mathematical Imaging and Vision, vol. 28, no. 2, pp. 179-190, 2007.

[28] S. Georgiev and J. Morais, "Bochner's theorems in the framework of quaternion analysis," in Quaternion and Clifford-Fourier Transforms and Wavelets, E. Hitzer and S. Sangwine, Eds., Trends in Mathematics, pp. 85-104, Springer, Berlin, Germany, 2013.

[29] S. Georgiev, J. Morais, K. I. Kou, and W. Sprößig, "Bochnerminlos theorem and quaternion fourier transform," in Quaternion and Clifford-Fourier Transforms and Wavelets, E. Hitzer and S. Sangwine, Eds., Trends in Mathematics, pp. 105-120, Springer, Berlin, Germany, 2013. 
[30] S. A. Collins, "Lens-system diffraction integral written in terms of matrix optics," The Journal of the Optical Society of America, vol. 60, pp. 1168-1177, 1970.

[31] M. Moshinsky and C. Quesne, "Linear canonical transformations and their unitary representations," Journal of Mathematical Physics, vol. 12, pp. 1772-1783, 1971.

[32] Y. L. Liu, K. I. Kou, and I. T. Ho, "New sampling formulae for non-bandlimited signals associated with linear canonical transform and nonlinear Fourier atoms," Signal Processing, vol. 90, no. 3, pp. 933-945, 2010.

[33] K. B. Wolf, Integral Transforms in Science and Engineering, vol. 11, chapter 9: canonical transforms, Plenum Press, New York, NY, USA, 1979.

[34] K. Gröchenig, Foundations of Time-Frequency Analysis, Birkhäuser, Boston, Mass, USA, 2001.

[35] Z. Zalevsky, D. Mendlovic, M. Alper Kutay, H. M. Ozaktas, and J. Solomon, "Improved acoustic signals discrimination using fractional Fourier transform based phase-space representations," Optics Communications, vol. 190, no. 1-6, pp. 95-101, 2001.

[36] K. I. Kou and R. H. Xu, "Windowed linear canonical transform and its applications," Signal Processing, vol. 92, no. 1, pp. 179-188, 2012.

[37] K. I. Kou, R. H. Xu, and Y. H. Zhang, "Paley-Wiener theorems and uncertainty principles for the windowned linear canonical transform," Mathematical Methods in the Applied Sciences, vol. 35, no. 17, pp. 2022-2132, 2012.

[38] K. Kou, J. Morais, and Y. Zhang, "Generalized prolate spheroidal wave functions for offset linear canonical transform in Clifford analysis," Mathematical Methods in the Applied Sciences, 2012.

[39] F. Brackx, R. Delanghe, and F. Sommen, Clifford Analysis, vol. 76, Pitman, Boston, Mass, USA, 1982.

[40] K.-I. Kou and T. Qian, "The Paley-Wiener theorem in $\mathbb{R}^{n}$ with the Clifford analysis setting," Journal of Functional Analysis, vol. 189, no. 1, pp. 227-241, 2002.

[41] A. Koç, H. M. Ozaktas, C. Candan, and M. A. Kutay, "Digital computation of linear canonical transforms," IEEE Transactions on Signal Processing, vol. 56, no. 6, pp. 2383-2394, 2008.

[42] R. Tao, B. Deng, and Y. Wang, Fractional Fourier Transform and Its Applications, Tsinghua University Press, Beijing, China, 2009.

[43] S. Abe and J. T. Sheridan, "Optical operations on wave functions as the Abelian subgroups of the special affine Fourier transformation," Optics Letters, vol. 19, no. 22, pp. 1801-1803, 1994.

[44] S.-C. Pei and J.-J. Ding, "Eigenfunctions of the offset Fourier, fractional Fourier, and linear canonical transforms," Journal of the Optical Society of America A, vol. 20, no. 3, pp. 522-532, 2003.

[45] K. K. Sharma and S. D. Joshi, "Uncertainty principle for real signals in the linear canonical transform domains," IEEE Transactions on Signal Processing, vol. 56, no. 7, part 1, pp. 2677-2683, 2008.

[46] D. Wei, Q. Ran, Y. Li, J. Ma, and L. Tan, "A convolution and product theorem for the linear canonical transform," IEEE Signal Processing Letters, vol. 16, no. 10, pp. 853-856, 2009.

[47] Y. Fu and L. Li, "Generalized analytic signal associated with linear canonical transform," Optics Communications, vol. 281, no. 6, pp. 1468-1472, 2008.

[48] B. M. Hennelly and J. T. Sheridan, "Fast numerical algorithm for the linear canonical transform," Journal of the Optical Society of America A, vol. 22, no. 5, pp. 928-937, 2005.
[49] K. K. Sharma and S. D. Joshi, "Signal separation using linear canonical and fractional Fourier transforms," Optics Communications, vol. 265, no. 2, pp. 454-460, 2006.

[50] P. Korn, "Some uncertainty principles for time-frequency transforms of the Cohen class," IEEE Transactions on Signal Processing, vol. 53, no. 12, pp. 523-527, 2005.

[51] E. M. S. Hitzer and B. Mawardi, "Uncertainty principle for the Clifford geometric algebra $\mathrm{Cl}_{n, 0}, n=3(\bmod 4)$ based on Clifford Fourier transform," in Wavelet Analysis and Applications, T. Qian, M. I. Vai, and Y. Xu, Eds., Applied and Numerical Harmonic Analysis, pp. 45-54, Springer, Berlin, Germany, 2006.

[52] E. M. S. Hitzer and B. Mawardi, "Clifford fourier transform on multivector fields and uncertainty principles for dimensions $n=$ $2(\bmod 4)$ and $n=3(\bmod 4), "$ Advances in Applied Clifford Algebras, vol. 18, no. 3-4, pp. 715-736, 2008.

[53] B. Mawardi and E. M. S. Hitzer, "Clifford Fourier transformation and uncertainty principle for the Clifford geometric algebra $\mathrm{Cl}_{3,0}$, " Advances in Applied Clifford Algebras, vol. 16, no. 1, pp. 41-61, 2006.

[54] M. Bahri and E. S. M. Hitzer, "Clifford algebra $\mathrm{Cl}_{3,0}$-valued wavelet transformation, Clifford wavelet uncertainty inequality and Clifford Gabor wavelets," International Journal of Wavelets, Multiresolution and Information Processing, vol. 5, no. 6, pp. 997-1019, 2007.

[55] E. Bayro-Corrochano, "The theory and use of the quaternion wavelet transform," Journal of Mathematical Imaging and Vision, vol. 24, no. 1, pp. 19-35, 2006.

[56] F. Brackx, N. De Schepper, and F. Sommen, "The two-dimensional Clifford-Fourier transform," Journal of Mathematical Imaging and Vision, vol. 26, no. 1-2, pp. 5-18, 2006. 


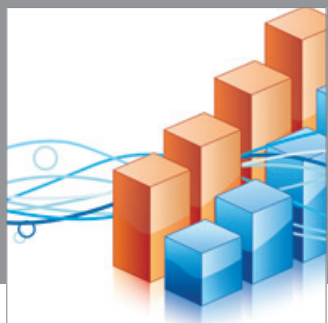

Advances in

Operations Research

mansans

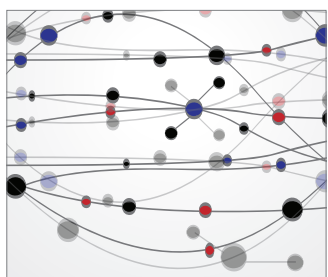

The Scientific World Journal
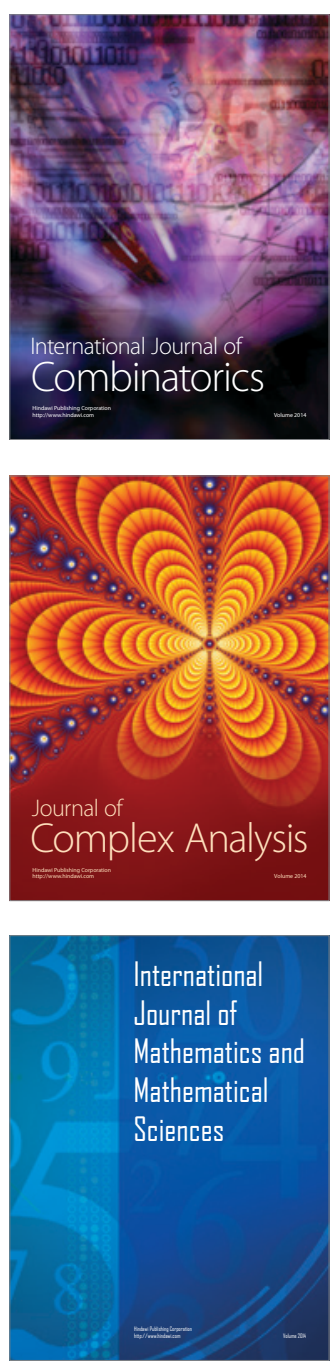
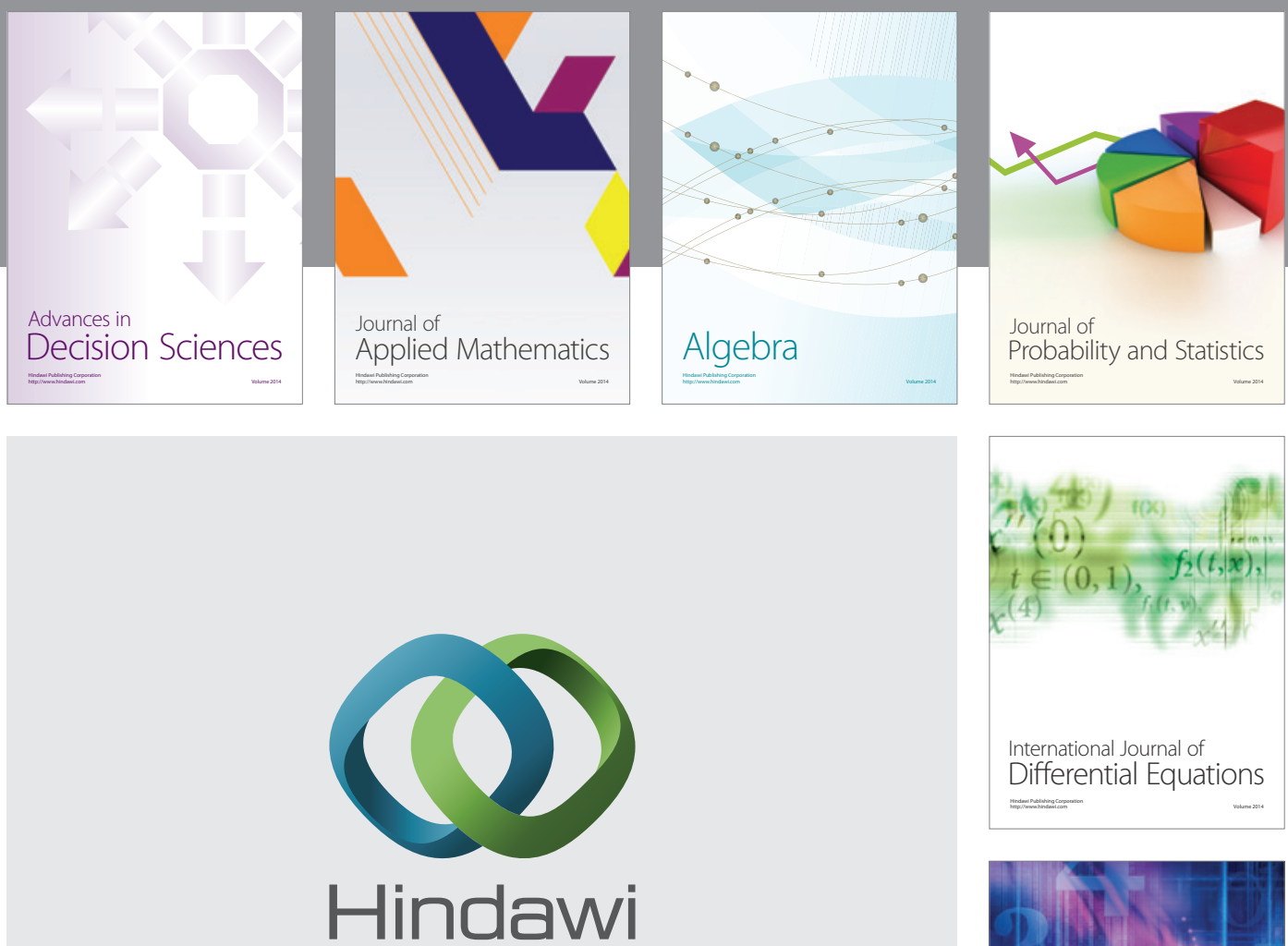

Submit your manuscripts at http://www.hindawi.com
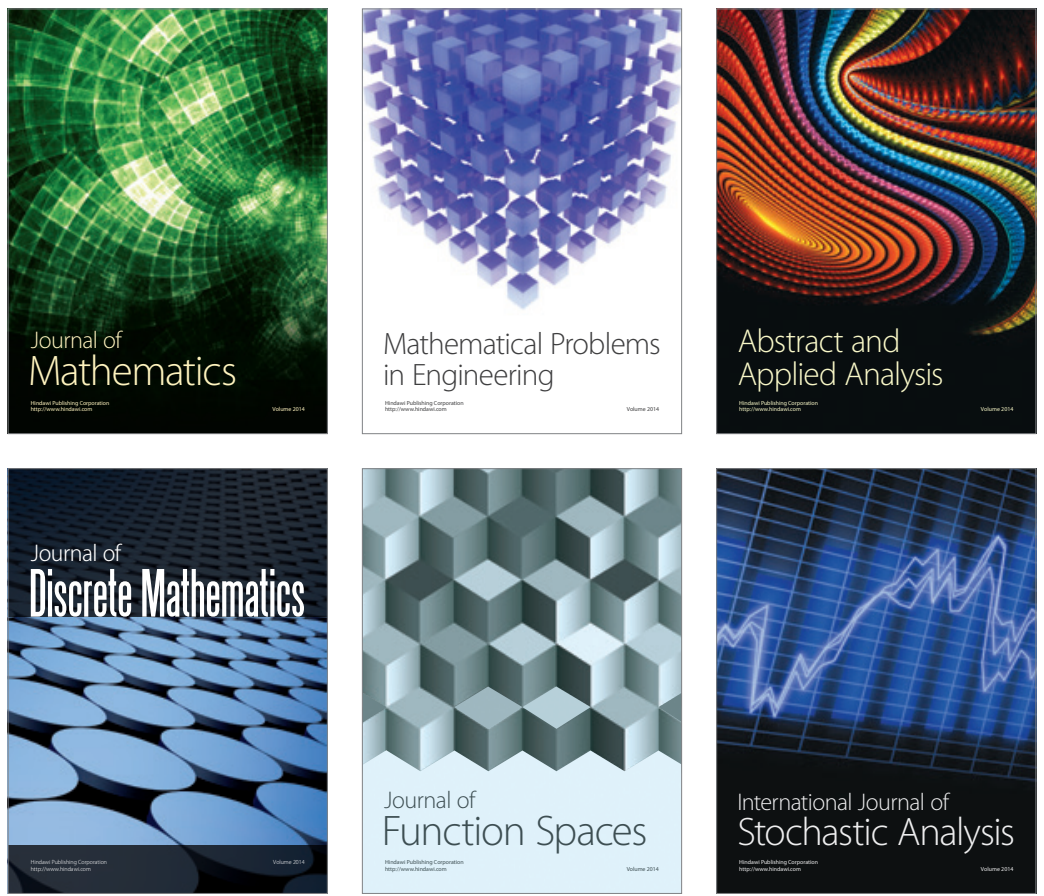

Journal of

Function Spaces

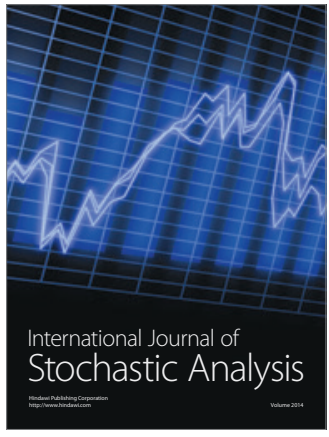

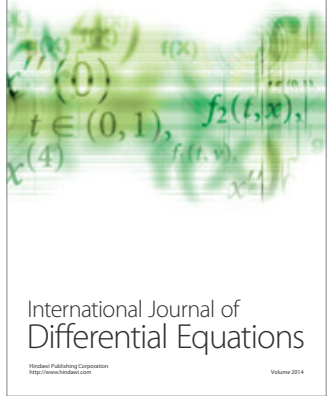
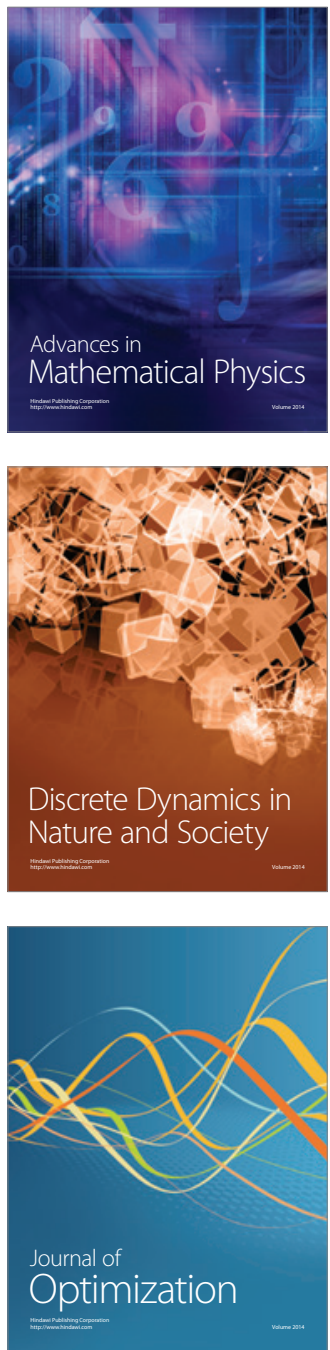